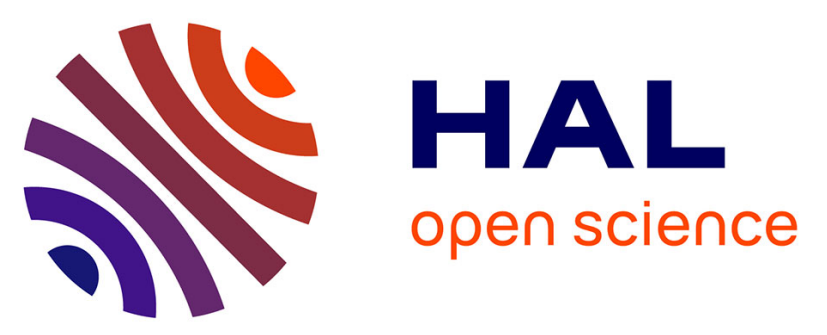

\title{
Analyse multi-méthodes de la déstabilisation d'un pylône de remontée mécanique implanté sur un glacier rocheux des Alpes françaises
}

Pierre-Allain Duvillard, Ludovic Ravanel, Philippe Schoeneich, Marco Marcer, Jean-François Piard

\section{To cite this version:}

Pierre-Allain Duvillard, Ludovic Ravanel, Philippe Schoeneich, Marco Marcer, Jean-François Piard. Analyse multi-méthodes de la déstabilisation d'un pylône de remontée mécanique implanté sur un glacier rocheux des Alpes françaises. Géomorphologie: relief, processus, environnement, 2019, 25 (1), pp.21-36. 10.4000/geomorphologie.12945 . hal-02350713

\section{HAL Id: hal-02350713 \\ https://hal.science/hal-02350713}

Submitted on 6 Nov 2019

HAL is a multi-disciplinary open access archive for the deposit and dissemination of scientific research documents, whether they are published or not. The documents may come from teaching and research institutions in France or abroad, or from public or private research centers.
L'archive ouverte pluridisciplinaire HAL, est destinée au dépôt et à la diffusion de documents scientifiques de niveau recherche, publiés ou non, émanant des établissements d'enseignement et de recherche français ou étrangers, des laboratoires publics ou privés. 


\title{
Analyse multi-méthodes de la déstabilisation d'un pylône de remontée mécanique implanté sur un glacier rocheux des Alpes françaises
}

\section{Multi-method analysis of the destabilization of a mechanical lift tower located on a rock glacier in the French Alps}

\author{
Pierre-Allain Duvillard ${ }^{*}$, Ludovic Ravanel a, Philippe Schoeneich ${ }^{c}$, Marco Marcer ${ }^{c}$, Jean-François Piard ${ }^{\mathrm{d}}$ \\ a Université Savoie Mont-Blanc, CNRS, EDYTEM UMR 5204 - Bâtiment « Pôle Montagne », 5 bd de la mer Caspienne, 73376 Le Bourget du Lac cedex, Chambéry, France. \\ ${ }^{\mathrm{b}}$ IMSRN, Parc Pré Millet - 680 Rue Aristide Berges, 38330 Montbonnot-Saint-Martin, France. \\ ${ }^{\text {c } U n i v e r s i t e ́ ~ G r e n o b l e ~ A l p e s, ~ I n s t i t u t ~ d ’ U r b a n i s m e ~ e t ~ d e ~ G e ́ o g r a p h i e ~ A l p i n e, ~ P A C T E ~-~ 14-14 b i s ~ a v e n u e ~ M a r i e ~ R e y n o a r d, ~} 38100$ Grenoble, France. \\ d SETAM - Val Thorens, 73440 Saint-Martin-de-Belleville, France.
}

INFORMATION SUR L'ARTICLE

Reçu le 18 juillet 2018

Reçu sous sa forme révisée le 28 janvier 2019

Définitivement accepté le 04 mars 2019

*Auteur correspondant. Tel : +33 (0)4 79758707 Courriels :

pierre-allain.duvillard@univ-smb.fr

(P-A. Duvillard)

ludovic.ravanel@univ-smb.fr (L. Ravanel)

philippe.schoeneich@univ-grenoble-alpes.fr

(P. Schoeneich)

marco.marcer@univ-grenoble-alpes.fr

(M. Marcer)

jfpiard@valthorens.com (J-F. Piard)

\begin{abstract}
RÉSUMÉ
La station de Val Thorens (Savoie, France) concentre plus de 79 composantes de remontées mécaniques en contexte de permafrost probable. Au cours de la dernière décennie, trois d’entre elles (deux gares amont et un pylône), situées vers $3000 \mathrm{~m}$ d'altitude, ont subi des déstabilisations partielles nécessitant d'importants travaux de confortement et de stabilisation. Cet article documente la déstabilisation du pylône $\mathrm{n}^{\circ} 2$ du funitel de Thorens au cours de lété 2016. Ses fondations ont été affectées par un déplacement horizontal et un affaissement à l'origine d'un décalage métrique de la tête de pylône par rapport au câble. Le pylône en question est implanté sur un glacier rocheux terrassé dans le cadre de l'aménagement d'une piste de ski. Une analyse multi-méthode a été conduite afin d'évaluer le rôle du permafrost dans la déstabilisation et de caractériser l'évolution des processus ainsi que l'impact des modifications anthropiques sur la dynamique du glacier rocheux à travers ( $i$ ) l’analyse stratigraphique de données de forages, (ii) une analyse du terrain porteur fondée sur des profils géophysiques de résistivité des matériaux acquis par tomographie électrique, et (iii) la reconstitution des modifications géomorphologiques de surface par comparaison de modèles numériques de terrain, obtenus par photogrammétrie numérique (images aériennes IGN et drone) avant et après terrassement. Cette étude met en évidence le rôle préparatoire du terrassement et des écoulements d'eau de surface mal contrôlés dans un milieu riche en glace tel qu'un glacier rocheux.
\end{abstract}

Mots clés : Remontées mécaniques, permafrost, glacier rocheux, subsidence, tomographie électrique résistive, Alpes françaises.

\section{ABSTRACT}

In the Val Thorens ski resort (Savoie, France), more than 79 infrastructure elements related to ropeway transport systems are located on permafrost. During the last decade, three of them (two top stations and a pylon located around 3,000 $m$ a.s.l.) experienced destabilization which required important adjustment and stabilization work. This study investigates the destabilization of the pylon \#2 of the Thorens funitel that occurred during the Summer 2016. Its foundations were affected by horizontal displacement and subsidence, causing a metric shift of the pylon top with respect to the cable. The pylon was built on a rock glacier which morphology was severely altered by earth work aiming to develop a ski slope. A multidisciplinary analysis was conducted in order to understand the processes that led to the pylon destabilization with a focus on the role of the morphological alterations of anthropogenic origin on the rock glacier dynamic. We used (i) a stratigraphic data analysis from boreholes and (ii) a subsurface analysis based on geophysics (Electrical Resistivity Tomography profiles) to understand the rock glacier internal structure, while (iii) geomorphological surface modifications were investigated by comparing digital terrain models obtained by aerial photogrammetry before and after the earthwork. This study highlights the preparatory role of the earthwork and a non-control of the surface water flow in an ice-rich environment such as a rock glacier.

Keywords: Ropeway transport systems, permafrost, rock glacier, subsidence, Electrical Resitivity Tomography, French Alps.

\section{Introduction}

Dans le contexte actuel de changement climatique, la haute montagne alpine est affectée par des changements majeurs tels que le retrait des glaciers et la dégradation (réchauffement) du permafrost (Beniston et al., 2018). Le permafrost est défini comme tous matériaux lithosphériques (sols, substratum rocheux, formations superficielles) dont les températures sont durablement inférieures ou égales à $0^{\circ} \mathrm{C}$ (Dobiński, 2011). Cette dégradation du permafrost est à l'origine d'une intensification potentielle des processus géomorphologiques (i.e., éboulements, écroulements, glissements, fluages) et de défis géotechniques (Harris et al., 2009). 
Les glaciers rocheux témoignent, sur le long terme, de conditions favorables pour la mise en place et le maintien du permafrost en montagne (Jones et al., 2019). Ces conditions semblent se maintenir depuis le début de l'Holocène, soit environ $10 \mathrm{Ka}$ (Krainer et al., 2015). Pour cette raison, ils représentent des éléments-clés dans la compréhension de l'évolution actuelle du permafrost dans le contexte actuel de réchauffement climatique (Haeberli et al., 2006). Dans les Alpes, une accélération du mouvement des glaciers rocheux a été observée au cours de ces deux dernières décennies (Delaloye et al., 2010 ; PERMOS, 2016). Les changements de température affectent la rhéologie de la glace et seraient responsables d'une augmentation de la déformation en raison d'une augmentation de la teneur en eau (Kääb et al., 2007 ; Ikeda et al., 2008 ; Cicoira et al., 2018). Les accélérations saisonnières pourraient aussi s'expliquer par des changements rapides (en quelques jours) dans l'hydrologie de surface, associés à des événements pluvieux intenses et/ou une fonte rapide de la neige (Wirz et al., 2017). L'infiltration d'eau peut devenir le principal facteur de mouvement et de déformation des glaciers rocheux en créant un horizon de cisaillement saturé en eau qui devient prépondérant dans l'écoulement du glacier rocheux ; cela représenterait $90 \%$ du mouvement par glissement et seulement $10 \%$ par fluage d'après Kenner et al. (2018). Cette dynamique caractérise les glaciers rocheux déstabilisés (Roer et al., 2008) et peut mener à une déstabilisation voire à un effondrement de sa partie frontale si les conditions topographiques le permettent (Bodin et al., 2016).

La déformation lente ou rapide des glaciers rocheux peut entraîner un risque de déstabilisation d'infrastructures construites directement sur ces derniers comme les remontées mécaniques, les refuges, ou les routes (Haeberli, 1992 ; Arenson et Jakob, 2017). Les éléments d'infrastructures (i.e., pylônes de remontées mécaniques) directement implantés sur des glaciers rocheux peuvent ainsi nécessiter un système d'ajustement progressif pour compenser les déplacements (Phillips et al., 2007 ; Arenson et al., 2009). Dans les Alpes françaises, le croisement d'un inventaire des infrastructures avec la carte d'indice de présence de permafrost (Permafrost Favourability Index) (Marcer et al., 2017) a permis d'identifier 947 éléments d'infrastructure (i.e., pylônes ou gares de remontées mécaniques, bâtiments, exploseurs à gaz) situés en contexte de permafrost potentiel, dont 20 sont construits sur des glaciers rocheux (Duvillard et al., soumis). La station de Val Thorens située en Savoie, est la deuxième station qui concentre le plus d'éléments d'infrastructures (plus de 148 dont 79 éléments de remontées mécaniques) en contexte de permafrost potentiel. Au cours de la dernière décennie, trois éléments d'infrastructures (deux gares d'arrivée et un pylône), construits vers $3000 \mathrm{~m}$ d'altitude, ont subi une déstabilisation nécessitant dans chacun des cas d'importants travaux de confortement et de stabilisation (Duvillard et al., sous presse).

L'impact de modifications anthropiques, telles que le remaniement des matériaux de surface pour l'installation d'une infrastructure et la création de pistes de ski, sur l'évolution et la dynamique des glaciers rocheux a été peu étudié. Dans les Alpes, seuls quelques glaciers rocheux aménagés ont été étudiés, comme le Ritigraben dans le Valais (Suisse ; Lugon et al., 2010 ; Kenner et al., 2017), Plan Bouchet en Vanoise (France) et Bellecombes dans les Écrins (France) (Bodin et al., 2010 ; Schoeneich et al., 2011), mais aucune étude n'a été menée sur les liens entre les modifications anthropiques du terrain et le déclenchement d'une instabilité pouvant affecter une infrastructure. Il existe néanmoins quelques études traitant d'instabilités dans des formations superficielles à proximité d'infrastructures, notamment dans les moraines à cœur de glace (Ravanel et al., 2018), de déstabilisations et de solutions de confortement d'infrastructures (Phillips et al., 2007).

La déstabilisation d'une infrastructure entraîne des surcoûts importants pour les gestionnaires, qui sont par ailleurs souvent largement démunis par rapport à ces problématiques nouvelles. Malgré des préconisations géotechniques de construction et de maintenance, certaines infrastructures sont affectées par des instabilités du fait de processus et facteurs d'instabilité sousestimés voire mal connus comme la modification des écoulements de surface. La compréhension de la complexité et de la réalité du terrain requiert des études de cas pratiques dans différents contextes géomorphologiques.

Cette recherche propose de documenter la déstabilisation du pylône $\mathrm{n}^{\circ} 2 \mathrm{du}$ funitel de Thorens au cours de l'été 2016, afin d'identifier les principaux facteurs préparatoires et déclencheurs de l'instabilité tout en montrant la part des forçages "naturels » (i.e., réchauffement climatique) et anthropiques (aménagement et inadéquations des préconisations techniques de construction). Cet article (i) décrit le site d'étude et présente les différentes étapes d'aménagement du glacier rocheux jusqu'à l'instabilité du pylône en juillet 2016, (ii) présente les méthodes utilisées pour caractériser l'évolution topographique avant/après aménagement et la distribution du permafrost grâce à des forages et des investigations géophysiques. Puis, (iii) les résultats permettront de caractériser la distribution du permafrost sous le pylône et mettront en évidence le rôle de l'eau dans le déclenchement de l'instabilité. Enfin, (iv) les investigations locales de terrain seront replacées dans le contexte climato-météorologique des dernières années et les choix quant au suivi géotechnique seront discutés.

\section{Un glacier rocheux fortement anthropisé}

\subsection{Le glacier rocheux de Thorens}

Présent sur le versant nord de la Pointe de Thorens entre $2800 \mathrm{~m}$ et $2950 \mathrm{~m}$ d'altitude, le glacier rocheux de Thorens est situé sur le domaine skiable de Val Thorens (massif de la Vanoise, France) (fig. 1). Il est constitué de deux lobes d'environ $60 \mathrm{~m}$ de large et $250 \mathrm{~m}$ de long composés de débris de roches sédimentaires de type grès et schistes argileux du houiller avec graphite (Guillot, 1982 ; Debelmas, 1989) (fig. 2). L'arête rocheuse située à l'Est se prolonge sous le front du lobe Est du glacier rocheux, formant un barrage naturel au fluage de celui-ci et à l'écoulement des eaux. Un lac temporaire - contraint par le glacier rocheux et cette arête rocheuse - est présent immédiatement à l'Est du glacier rocheux. La carte d'indice de dégradation du permafrost (Potentially Thawing Permafrost - PTP) (Marcer et al., 2019.), qui propose une distribution spatiale du permafrost en cours de dégradation dans les Alpes françaises, indique un PTP de 0,95. le glacier rocheux est situé dans une zone à " permafrost subissant une dégradation importante » provoquée par l'augmentation de la température moyenne annuelle de l'air. 

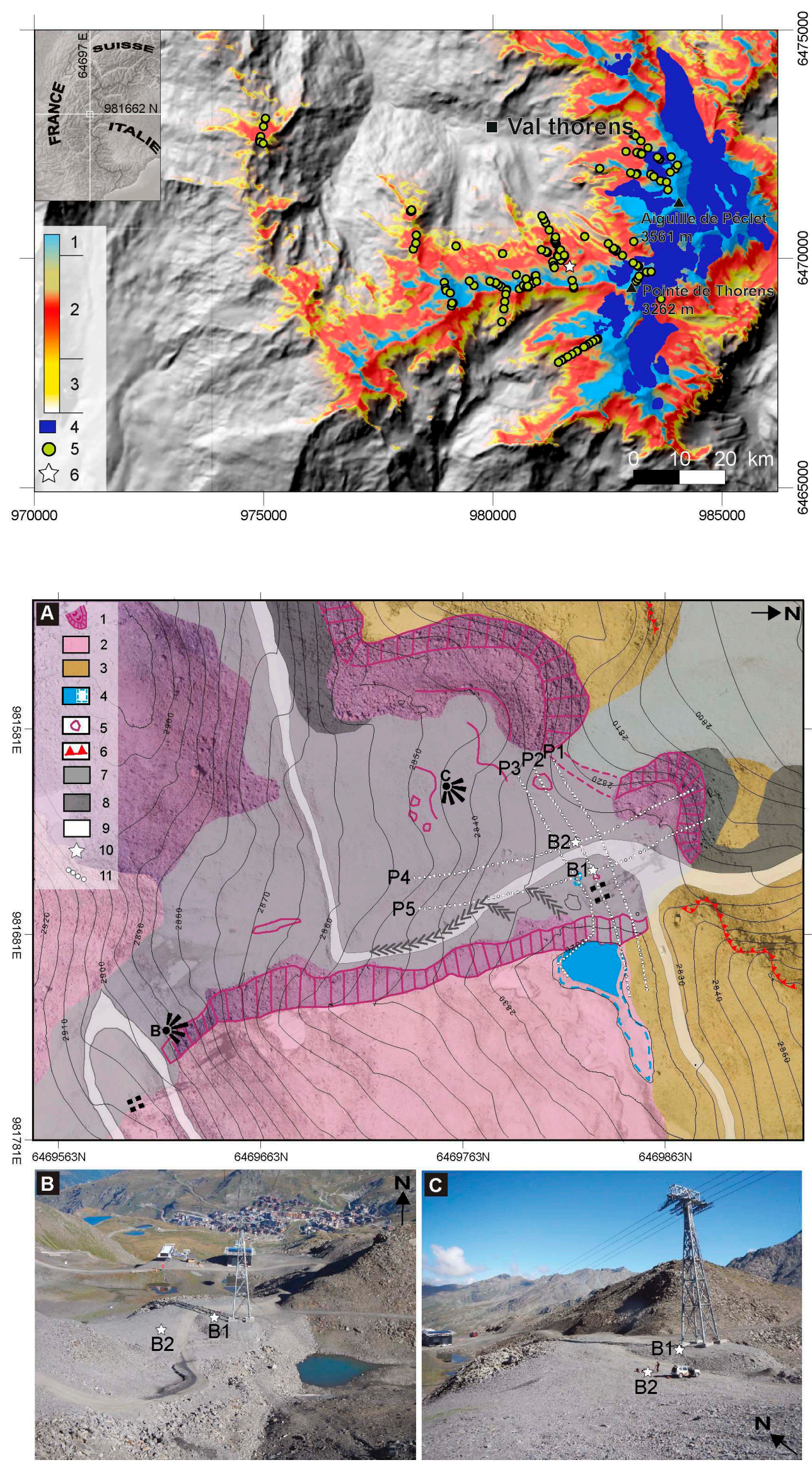

8
0
0
0
0

Fig. 1 - Carte de localisation des remontées mécaniques de Val Thorens, du pylône $n^{\circ} \mathbf{2}$ du funitel de Thorens et du permafrost.

Extension des glaciers d'après Gardent et al (2014) ; modélisation du permafrost en cours de dégradation d'après Marcer et al. (2019). 1. Permafrost; 2. Permafrost 8 en cours de dégradation ; 3. Présence improbable de permafrost ; 4. Extension des glaciers en 2009 ; 5. Infrastructures ; 6. Pylône $\mathrm{n}^{\circ} 2$ du funitel de Thorens.

Fig. 1 - Location map of the Val Thorens ski lifts and pylon \#2 of Thorens funitel compared to permafrost.

Extension of glaciers according to Gardent et al. (2014); map of the permafrost undergoing degradation according to Marcer et al. (2019). 1. Permafrost; 2. Thawing permafrost; 3. Improbable permafrost; 4. Glacier extent 8 in 2009; 5. Infrastructure; 6. Pylon \#2 of the Thorens funitel.

Fig. 2 - Géomorphologie du site d'étude. A : Croquis géomorphologique de la zone d'étude (utilisation de la légende UNIL Lambiel et al., 2013). B : Vue vers le Nord du glacier rocheux en septembre 2016. C : Vue en direction du lobe nord-est du glacier rocheux en septembre 2016. 1. Glacier rocheux inactif 2. Accumulation périglaciaire; 3 . Substratum rocheux; 4. Lac / lac temporaire ou zone d'infiltration ; 5. Subsidence thermokarstique ; 6. Escarpement rocheux ; 7. Surface terrassée ; 8. Dépôts d'origine anthropique ; 9. Piste 10. Forage; 11. Profil géoélectrique; 12. Renvoi d'eau.

Fig. 2-Geomorphological characteristics of the study site.

A: Geomorphological sketch of the study area. B: North view of the rock glacier in September 2016. C: View towards the north-east lobe of the rock glacier in September 2016. 1. Inactive rock glacier; 2. Periglacial scree slope; 3. Bedrock; 4. Lake / ephemeral lake or water infiltration area; 5 . Thermokarst; 6 . Rock scarp 7. Earthwork; 8. Artificial deposits; 9. Track; 10. Borehole; 11. ERT; 12. Drain. 


\subsection{Laménagement du glacier rocheux de Thorens}

Le funitel de Thorens a été construit durant l'été 2011 pour environ $7 \mathrm{M} €$. Il est composé de deux gares et de quatre pylônes dont trois sont installés sur du permafrost probable (Duvillard et al., 2015). Le pylône $\mathrm{n}^{\circ} 2$ a été construit sur le lobe Est du glacier rocheux après une étude de faisabilité de 2008 et une étude géotechnique de 2010 (Fabre et al., 2015). La présence de permafrost et d'un secteur riche en glace dans le glacier rocheux a été signalée à l'occasion de ces études à partir de deux profils de tomographie de résistivité électrique (ERT). Ces études ont contribué au choix de l'implantation des fondations du pylône $\mathrm{n}^{\circ} 2$, en préconisant une implantation décalée en amont du front du glacier rocheux dans une zone avec « moins de glace » qu'au niveau du front. Le constructeur du funitel avait prévu une adaptation possible du pylône à l'aide d'un rail réglable, en cas de mouvement du glacier rocheux, mais uniquement dans le sens latéral sans basculement de la pente (est-ouest). Le pylône a également été fondé sur 4 appuis (semelles béton) sur une couche d'environ $2 \mathrm{~m}$ de gravier reposant elle-même sur le permafrost dans le but de favoriser une circulation de l'air sous les semelles et de recréer une couche active. Cette méthode est d'ailleurs préconisée par le guide "Construire sur le permafrost " (Bommer et al., 2010). Une plateforme de remblais d'environ $2 \mathrm{~m}$ d'épaisseur a également été créée pour recouvrir les fondations du pylône afin de limiter les variations de température et la conduction thermique dans le soussol.

Une piste de ski a par ailleurs été construite en 2011 sur le glacier rocheux après un travail de terrassement mobilisant le terrain sur plusieurs mètres d'épaisseur de manière à aplanir la surface du sol (fig. 3). Les plus gros blocs de surface ont été déplacés pour faciliter le damage par faible enneigement. En juin 2015, une piste pour véhicules tout-terrain a été construite en amont du pylône afin de faciliter l'accès à une zone de travaux située au col de Rosaël (2990 m).

\subsection{La déstabilisation du pylône $\mathrm{n}^{\circ} 2 \mathrm{du}$ funitel}

Alors qu'une anomalie de réglage du balancier du pylône $\mathrm{n}^{\circ} 2$ avait été identifiée au cours de l'automne 2015, le pylône a été affecté par un basculement à la fin du mois de juillet 2016. Un déplacement des fondations de $25 \mathrm{~cm}$ latéralement (est-ouest) et de $55 \mathrm{~cm}$ verticalement a été mesuré (donnée Topoalp). Ce mouvement a généré un décalage horizontal d'environ $1,5 \mathrm{~m}$ du sommet du pylône et par suite un très important désalignement du pylône par rapport aux câbles. Trois indices géomorphologiques d'activité ont été identifiés lors d'une visite de terrain réalisée début août 2016 (fig. 4) : (i) une subsidence de rayon métrique à proximité des fondations, (ii) des fissures formées en amont des fondations, et (iii) une zone d'infiltration avec un dépôt de sédiments fins et argileux en amont de la plateforme du pylône.

\section{Méthodes}

Quatre méthodes ont été mises en œuvre sur le site, afin de documenter et d'identifier l'origine de la déstabilisation : (i) la reconstitution topographique historique des modifications géomorphologiques de surface par comparaison de modèles numériques de terrain (MNT) obtenus par photogrammétrie aérienne (images d'avion IGN en 2009 et images par drone en 2017) et par un levé par balayage laser terrestre (TLS pour Terrestrial Laser Scanning)également réaliséen 2017; (ii)la comparaison diachronique de photographies terrestres ; (iii) des mesures géoélectriques le long de 5 profils en pseudo-3D pour mettre en évidence les contrastes et anomalies de résistivité ; et (iv) deux forages destructifs réalisés afin de valider la profondeur du substratum rocheux pour proposer une solution de confortement.

\subsection{Reconstitution topographique historique de la surface}

La photogrammétrie aérienne permet de reconstituer la topographie de surface et ainsi de mesurer ses changements à partir d'une
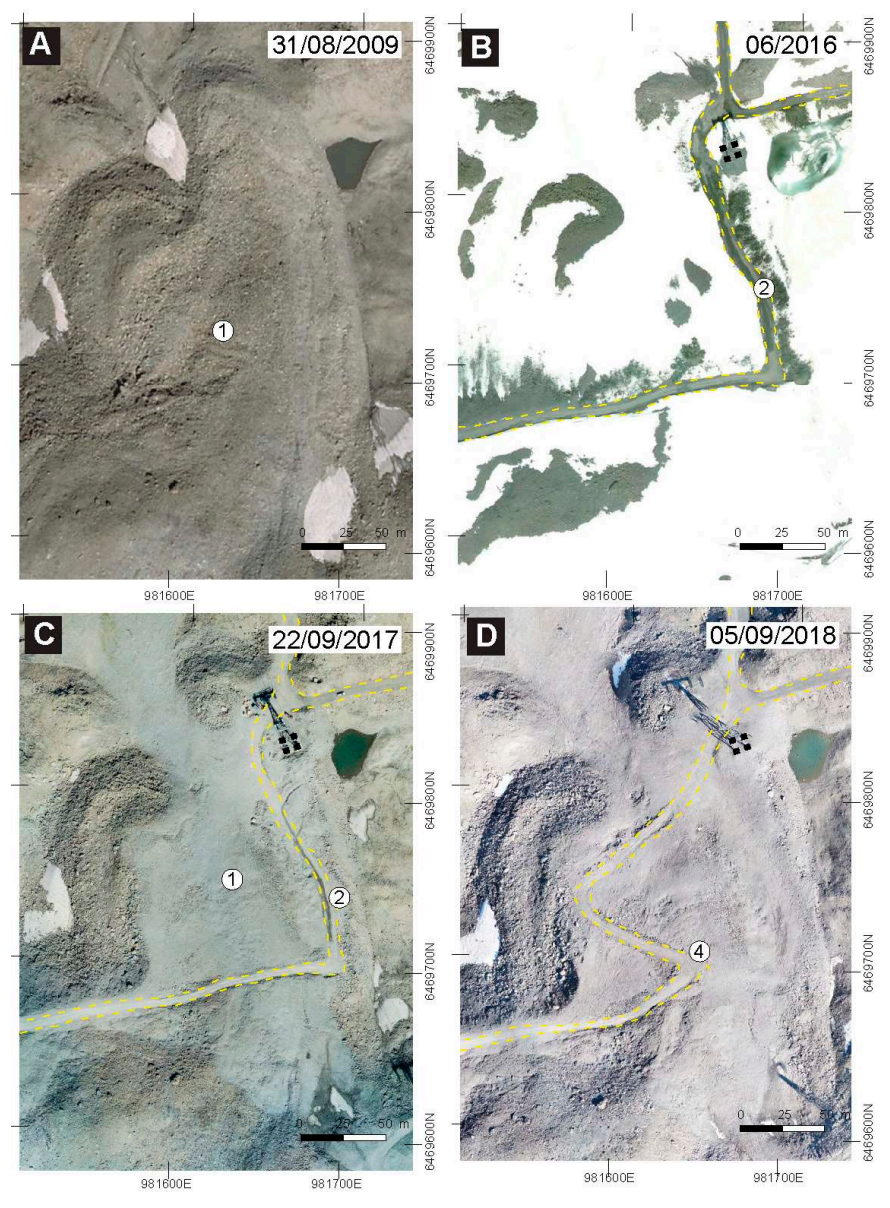

Fig. 3 - Évolution de la surface du glacier rocheux de Thorens entre 2009 et 2018 .

A : images aériennes IGN de 2009 avant l'aménagement du funitel et la création de la piste de ski. B : images IGN de juin 2016 avec déneigement précoce de la piste pour véhicule. C : images drone de 2017 assemblées. D : images drone de 2018 assemblées avec une modification d'emplacement de la piste pour véhicule au centre du glacier rocheux. 1. Piste de ski nivelée en 2011 ; 2. Piste pour véhicules construite en 2015 et déneigée artificiellement en juin 2016 pour permettre un accès à des travaux en amont ; 3. Piste pour véhicules construite en octobre 2017.

Fig. 3 - Evolution of the surface of the Thorens rock glacier between 2009 and 2018.

A: 2009 orthoimage obtained using aerial images before the building of the funitel and the ski slope (IGN). B: 2016 image of vehicle track artificially cleared of snow in June (IGN). C: 2017 orthoimage obtained using UAV images. D: 2018 orthoimage obtained using UAV images. 1. Ski slope created in 2011; 2. Vehicle track built in 2015 and artificially cleared of snow in June 2016 to allow access to work; 3. New track for vehicles sat up in October 2017 
analyse différentielle multi-dates des photographies acquises par drone, avion ou hélicoptère. Cette méthode a été très utilisée pour l'étude de glaciers rocheux dans la période récente (Kenner et al., 2014 ; Capt et al., 2016 ; Dall'Asta et al., 2017). Afin d'observer les changements morphologiques à la surface du glacier rocheux de
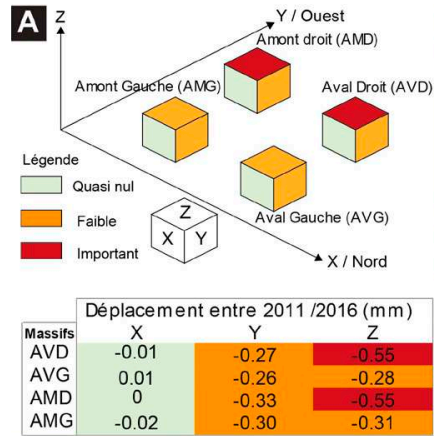

C

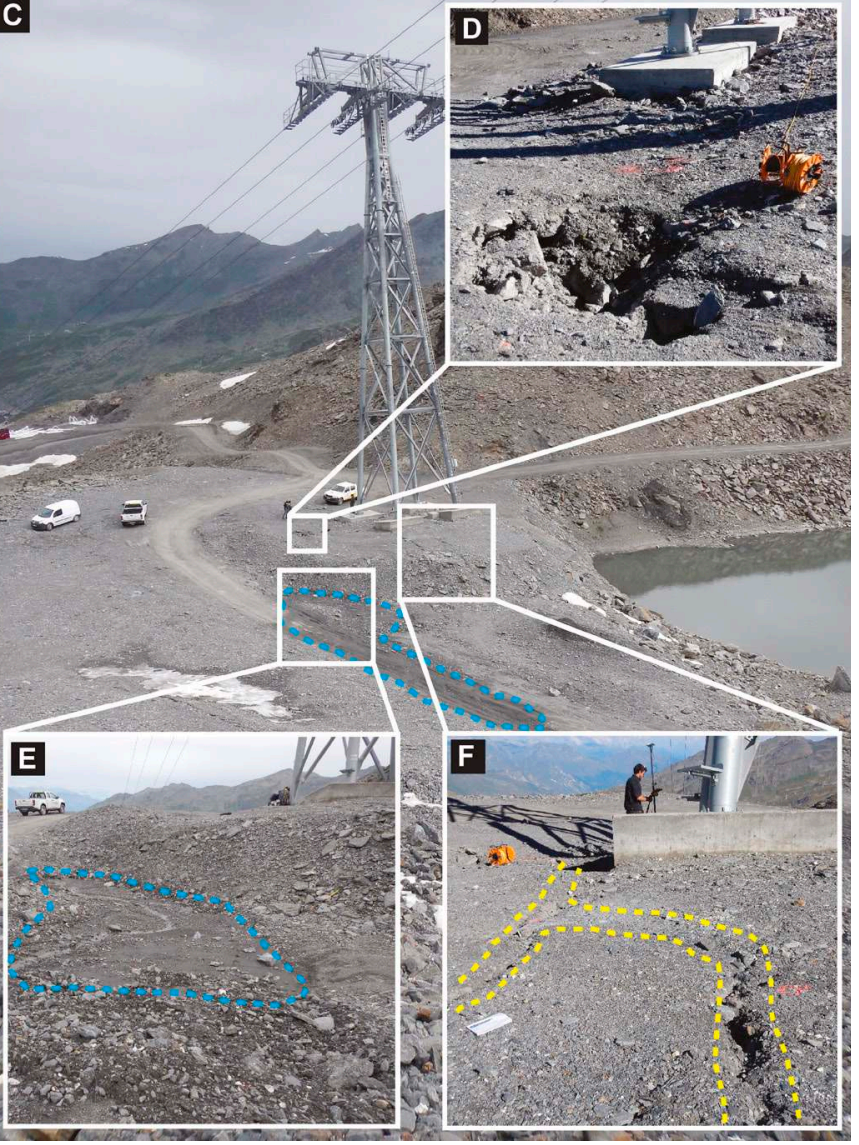

Fig. 4 - Schéma de l'affaissement des fondations et indices de déstabilisation du pylône $\mathrm{n}^{\circ} \mathbf{2}$ du funitel de Thorens en août/septembre 2016.

A : Mesures de déplacement des massifs du pylône entre avril 2011 et août 2016 B : Décalage de $1,47 \mathrm{~m}$ au sommet du pylône entre le câble et le balancier. C : Vue générale du pylône (vue SO vers le NE). D : Entonnoir de suffosion de rayon métrique à l'Ouest des fondations. E : Zone d'infiltration de l'eau en amont de la plateforme du pylône. F : Fractures de subsurface visibles en amont des fondations.

Fig. 4 - Scketch of the subsidence of foundations and destabilization signs on the pylon \#2 of the funitel de Thorens.

A: Movement survey of the pylon foundations between April 2011 and August 2016 B: Offset at the top of the pylon between the cable and the pendulum. C: General view of the pylon (view SW to NE). D: Metric subsidence West of the foundations. E: Water infiltration zone, upstream of the pylon platform. F: Subsurface fractures visible upstream of the foundations.
Thorens, deux MNT à maille de $1,5 \times 1,5 \mathrm{~m}$ ont été générés à partir de nuages de points photogrammétriques : (i) un modèle avant les travaux de terrassement à partir des images aériennes IGN acquises par avion en août 2009, (ii) un modèle post-instabilité réalisé à partir d'images de drone DJI Mavic Pro acquises en septembre 2017. Enfin, un vol drone avec un DJI Phantom 4 a été réalisé en septembre 2018 pour générer une orthophotographie du glacier rocheux après les travaux de 2017 (tab. 1).

Le worflow décrit par Smith et al. (2016) a été utilisé pour générer des nuages de points du glacier rocheux pour les deux années à l'aide d'Agisoft PhotoScan (v.1.2.6). Pour les images de 2009, un réseau de 22 Points de Contrôle au Sol (PCS) a été levé au niveau de points topographiques remarquables (gros blocs, pylônes, angles de terrasse de bâtiments, etc.). En 2017, 9 PCS sur cibles (bâches avec croix) ont été levés pour l'ortho-rectification des images. L'ensemble des PCS a été levé avec un GPS différentiel (dGPS) Trimble Geo7X d'une précision centimétrique.

Dans le but de garantir une bonne géométrie des nuages de points photogrammétriques, une acquisition par TLS a été réalisée à la même date, en 2017, avec un scanner Optech ILRIS 3D (2 000 pts.sec ${ }^{-1}$ pour une fenêtre de $40^{\circ} \times 40^{\circ}$ avec une portée maximale de $400 \mathrm{~m}$ ), depuis 4 positions avec un total de 13 scènes. Les nuages de points obtenus ont été alignés à l'aide du logiciel CloudCompare 2.10.V2 (Girardeau-Montaut, 2006). Une consolidation des alignements a été réalisée avec l'algorithme Iteractive Closest Point (ICP) pour les différentes positions (Porlemeleau et al., 2013). Enfin, les nuages obtenus par photogrammétrie ont été alignés sur les nuages TLS par la procédure ICP en vue d'améliorer la géométrie des nuages de points (Bodin et al., 2018). Des MNT de 1,5 $\mathrm{m}$ de résolution ont été générés à partir des nuages de points de 2009 et 2017 avec Cloud Compare, puis l'écart entre les surfaces a été calculé par soustraction avec SAGA GIS.

\subsection{Comparaison de photographies}

Le glacier rocheux a fait l'objet de deux visites de terrain en septembre 2014 puis en juillet 2015, c'est à dire avant l'instabilité de 2016, dans le cadre d'un inventaire des dommages sur les infrastructures de Val Thorens (Duvillard et al., 2015, sous presse). La comparaison des photographies terrestres à ces deux dates permet de documenter précisément l'évolution de la micro-topographie autour du pylône.

\subsection{Prospections géoélectriques dans la zone instable}

La prospection géoélectrique est une méthode couramment utilisée pour étudier le permafrost de montagne, en particulier au niveau des glaciers rocheux, en complément détudes géomorphologiques (Maurer et Hauck 2007 ; Scapozza et al., 2011a ; Springman et al., 2012 ; Scapozza et Laigre 2014). La résistivité électrique des matériaux augmente avec la teneur en glace et permet de distinguer les sédiments gelés de ceux non gelés (Scott et al., 1990 ; Kneisel et al., 2008).

Cinq profils ERT ont été réalisés fin août 2016, puis onze profils en juillet 2017 à la fois par ERT et par polarisation induite (IP) afin de caractériser précisément la distribution du permafrost en $3 \mathrm{D}$ dans l'ensemble du glacier rocheux. Les résultats sont détaillés dans Duvillard et al. (2018). Pour cet article, seuls les 5 profils réalisés 
Tab. 1 - Caractéristiques et précision des alignements des données topographiques.
Tab. 1 - Characteristics and resolution of the data used for the orthoimage and DEM reconstruction of the study site.

\begin{tabular}{lcccc}
\hline Dates & 31 août 2009 & & 22 sept. 2017 & 05 sept. 2018 \\
\hline Types d'acquisition & Avion & Drone & TLS & Drone \\
\hline Modèle & - & DJI Mavic Pro & Optech Ilris & DJI Phantom 4 \\
\hline Nb d'images / scènes & 5 & 174 & 13 & - \\
\hline Nb de points de PCS & 22 & 9 & 7000 & 7 \\
\hline Nb de points $\times 10^{3}$ & 106 & 1600 & 306 & - \\
\hline Densité de points (points $\left./ \mathrm{m}^{2}\right)$ & 6 & 1,4 & - & - \\
\hline $\begin{array}{l}\text { Erreur moyenne d'alignement sur le nuage } \\
\text { de point TLS 2017 en Z }(\mathrm{m})\end{array}$ & 1,8 & & & - \\
\hline
\end{tabular}

en septembre 2016, c'est-à-dire directement après l'instabilité, seront présentés et inversés en 2D. Les acquisitions par ERT ont été réalisées avec le résistivimètre multi-électrodes ABEM Terrameter SAS 4000 composé de 4 canaux pour un dispositif de 64 électrodes (fig. 2, tab. 2). L'écartement des électrodes était de $2 \mathrm{~m}$ pour les profils transversaux et de $2,5 \mathrm{~m}$ pour les profils longitudinaux en raison de la longueur de ces derniers. Le protocole Wenner avec 404 points de mesures a été utilisé pour obtenir le meilleur rapport signal/bruit. Ce protocole est sensible aux changements verticaux de structure et aux signaux faibles (Dahlin et Zhou 2004); il est couramment utilisé pour sa robustesse dans les études en contexte de permafrost (Kneisel, 2006). En cas de gros blocs en surface, le contact entre les électrodes et le sol pouvait être amélioré en utilisant de l'eau salée et de la bentonite. La position des électrodes a été levée avec un dGPS. Pour chaque profil ERT, le temps d'acquisition était de $0,3 \mathrm{~s}$ avec une temporisation de $0,5 \mathrm{~s}$. L'intensité de l'injection variait de 5 à $200 \mathrm{~m}$ selon la résistance du sous-sol. Pendant l'acquisition, si un point mesuré présentait une erreur Root Mean Square (RMS) > $1 \%$, la mesure pouvait être répétée jusqu'à 4 fois. Les données ont été inversées avec la version 3.54.44 du logiciel Res2Dinv (Loke et Barker, 1996) qui utilise la méthode des moindres carrés pour produire un modèle $2 \mathrm{D}$ du sous-sol à partir des résistivités apparentes mesurées. Les cinq profils ERT inversés utilisent la même échelle d'interprétation des résistivités que de précédents travaux sur des glaciers rocheux (Scapozza et al., 2011 ; Bosson et al., 2014).

Tab. 2 - Synthèse du traitement des profils d'ERT.

Tab. 2 - Synthesis of the processing of the ERT profiles.

\begin{tabular}{cccccc}
\hline $\mathrm{N}^{\circ}$ profiles ERT & P1 & P2 & P3 & P4 & P5 \\
\hline $\begin{array}{c}\text { Espacement entre les } \\
\text { électrodes }(\mathrm{m})\end{array}$ & 2 & 2 & 2 & 2,5 & 2,5 \\
\hline Nb de points mesures & 404 & 404 & 401 & 401 & 403 \\
\hline Nb d'itérations & 3 & 4 & 3 & 3 & 3 \\
\hline $\begin{array}{c}\text { Erreur moyenne } \\
\text { quadratique }\end{array}$ & 4,3 & 6,4 & 4,4 & 3,6 & 4,7 \\
\hline
\end{tabular}

\subsection{Forages destructifs}

Les forages destructifs sont une méthode directe pour déterminer une éventuelle stratification dans un corps gelé. Moins précis mais moins coûteux qu'un forage carotté, l'interprétation de la stratification repose notamment sur l'expérience du foreur (Arenson et al., 2002). S'il s'en suit une instrumentation, ils peuvent également être utilisés pour suivre l'évolution de la température en profondeur, en subsurface, ou la déformation. Dans les Alpes françaises et à des fins de recherche, il existe des forages pour le suivi des températures du permafrost en contexte de formations superficielles des glaciers rocheux en particulier (Bodin et al., 2015), de substratum rocheux (Schoeneich et al., 2015), et de parois rocheuses (Magnin et al., 2015).

Deux forages destructifs par marteau fond de trou avec enregistrement des paramètres ont été réalisés fin septembre 2016 dans l'objectif de valider la profondeur du substratum rocheux pour proposer une solution de confortement géotechnique. Ils ont été réalisés dans le lobe Est du glacier rocheux à proximité du pylône : le forage $\mathrm{B} 1$, d'une profondeur de $23,7 \mathrm{~m}$, au sein de la zone de subsidence, et le forage B2, d'une profondeur de $27,8 \mathrm{~m}$, à l'Ouest de la piste pour véhicule). Les deux forages ont été poursuivis une dizaine de mètres dans le substratum rocheux. La nature des matériaux traversés a été déterminée grâce à l'expérience de l'équipe de foreurs et à partir de l'observation des matériaux expulsés (cuttings). Une estimation qualitative de la présence de glace (présence/absence) a été établie sur la base du type et/ou de la texture des matériaux expulsés et de la résistance à la pénétration de la tête de forage (i.e., la Vitesse Instantanée d'Avancement).

\section{Résultats et interprétations}

Les facteurs préparatoires au déclenchement de l'instabilité sont présentés en trois parties : (i) l'évolution de la topographie et des écoulements de surface dans le secteur d'étude par comparaison des MNT de 2009 à 2017, complétée par la comparaison de photographies terrestres; (ii) l'interprétation des profils ERT du lobe Est du glacier rocheux ; et (iii) l'interprétation du profil transversal P2 - qui documente la stratification des matériaux rocheux présents sous le pylône - par comparaison avec la stratigraphie des forages.

\subsection{Remaniement des matériaux de surface et modification des écoulements}

La construction de la piste de ski et de la piste pour véhicules a largement modifié la micro-topographie du glacier rocheux ainsi que l'écoulement des eaux de surface. La comparaison des MNT 
générés en 2009 et 2017 permet de mettre en évidence des écarts positifs et négatifs de 2 à $6 \mathrm{~m}$ verticalement (fig. 5). La carte des pentes et les photos aériennes de 2017 montrent que cela correspond à un nivellement régulier de la surface (fig. 6A, 3B). En 2009, l'infiltration diffuse des eaux de surface dans le glacier rocheux était favorisée par la rugosité du terrain. Depuis les travaux de nivellement de 2011, la perméabilité de la surface a été réduite par fermeture des pores, favorisant ainsi le ruissellement.

L'aménagement en 2015 de la piste pour véhicules en amont du pylône a concentré le ruissellement en canalisant l'eau en amont du remblai du pylône (fig. 6B). En 2014, la partie amont du remblai n'était pas encore ou peu affectée par des écoulements de surface. Durant les étés 2015 et 2016, les écoulements de surface empruntaient la piste avant de s'infiltrer au niveau du remblai du pylône. Un fossé et un renvoi d'eau ont été creusés en août 2016 afin de canaliser et d'évacuer les écoulements d'eau vers le lac (fig. 6C).

Par ailleurs, la piste pour véhicules a été déneigée précocement au début de l'été 2016, afin que des véhicules et engins (camions, pelleteuses) puissent accéder à un chantier situé en amont, modifiant les débits et la circulation des eaux de surface en début d'été (fig. 3B).

\subsection{Un glacier rocheux poly-thermal}

Le lobe Est du glacier rocheux est divisé en deux parties spécifiques (fig. 7) : (i) une partie droite non gelée sous la plateforme du pylône, et (ii) une partie gauche gelée principalement dans le front du lobe et à la marge avec le lobe Ouest du glacier rocheux, au centre de la piste de ski aménagée. La résistivité de la zone gelée varie entre 10 et $55 \mathrm{k} \Omega \mathrm{m}$ et correspond à la présence d'un permafrost sous la forme de matériaux rocheux et de glace (i.e., ice/rock mixture). D’après l'échelle de Bosson et al. (2014), il pourrait s'agir d'un permafrost tempéré (température proche du point de fusion) et (sub)saturé en eau. Des valeurs semblables ont également été rencontrées dans des matériaux fins avec de la glace finement dispersée (Bodin et al. 2016). Ainsi, le pylône $n^{\circ} 2$ est implanté dans un secteur dont le permafrost est absent, mais à environ $5 \mathrm{~m}$ d'une zone de transition entre des matériaux gelés et non gelés où se situe précisément la subsidence.

Ainsi, la localisation de la zone d'infiltration observée en 2016 correspondrait à (i) la zone de transition entre la partie gelée et celle non gelée (i.e., transition imperméable/perméable) et

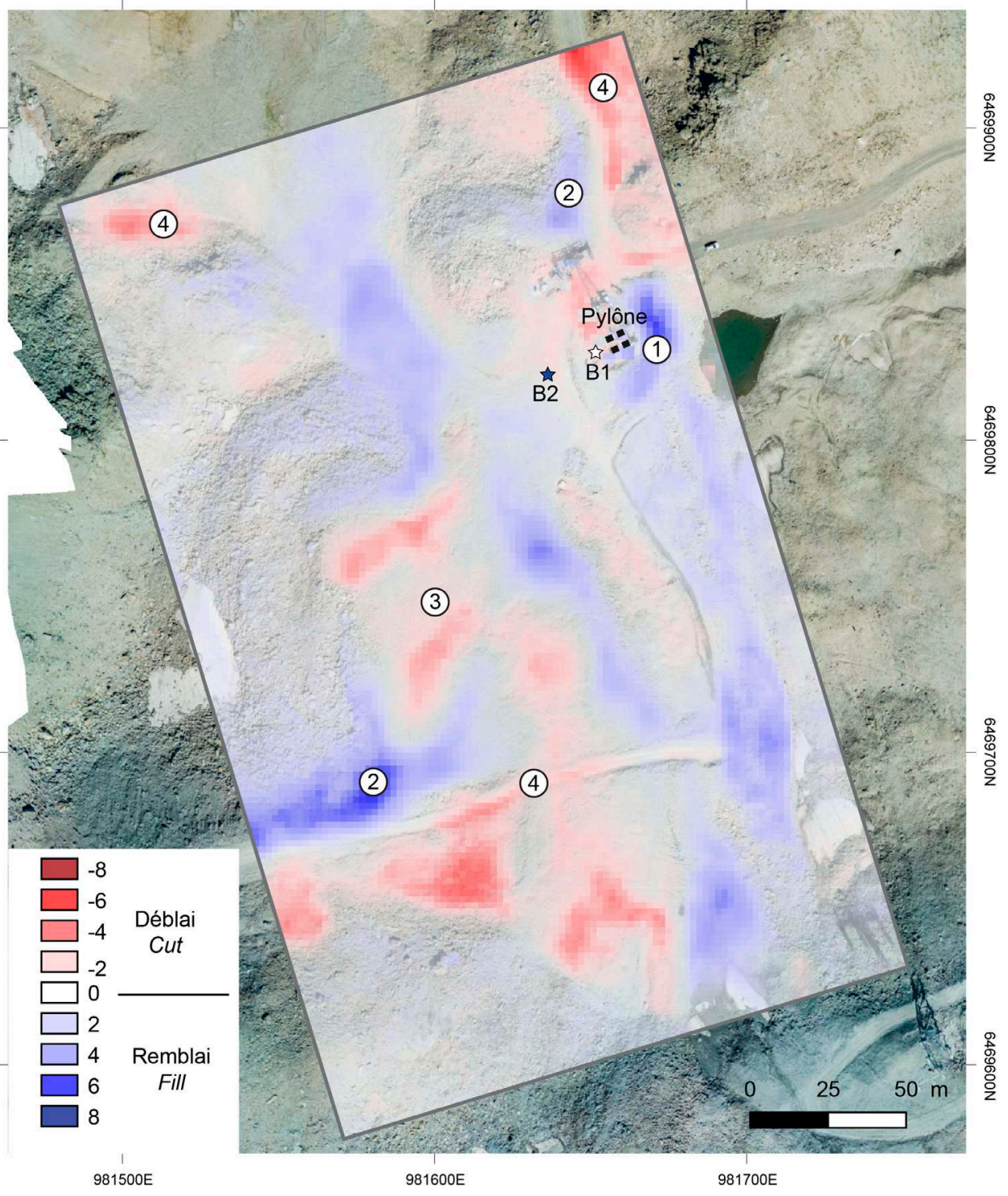

Fig. 5 - Carte des écarts d'altitude de la surface du glacier rocheux de Thorens entre 2009 et 2017.

1. Plateforme du pylône; 2 . Talus de dépôt de matériaux résultant de l'aménagement des pistes: 3. Aplanissement pour la création d'une piste de ski ; 4. Creusement pour la création d'une piste pour véhicules.

Fig. 5 - Surface elevation changes of the Thorens rock glacier between 2009 and 2017.

1. Platform of the pylon; 2. Talus slope of deposits from the creation of the tracks; 3. Smoothing for the creation of a ski slope; 4. Digging for the creation of the track. 
(ii) à la barrière topographique que constitue la plateforme du pylône. Il est à noter que le lac induit probablement un secteur dégelé permettant de maintenir hors permafrost cette partie du glacier rocheux. Les investigations géophysiques menées par Duvillard et al. (2018) confirment cette distribution.

\subsection{Un lessivage des matériaux fins au niveau des fondations du pylône}
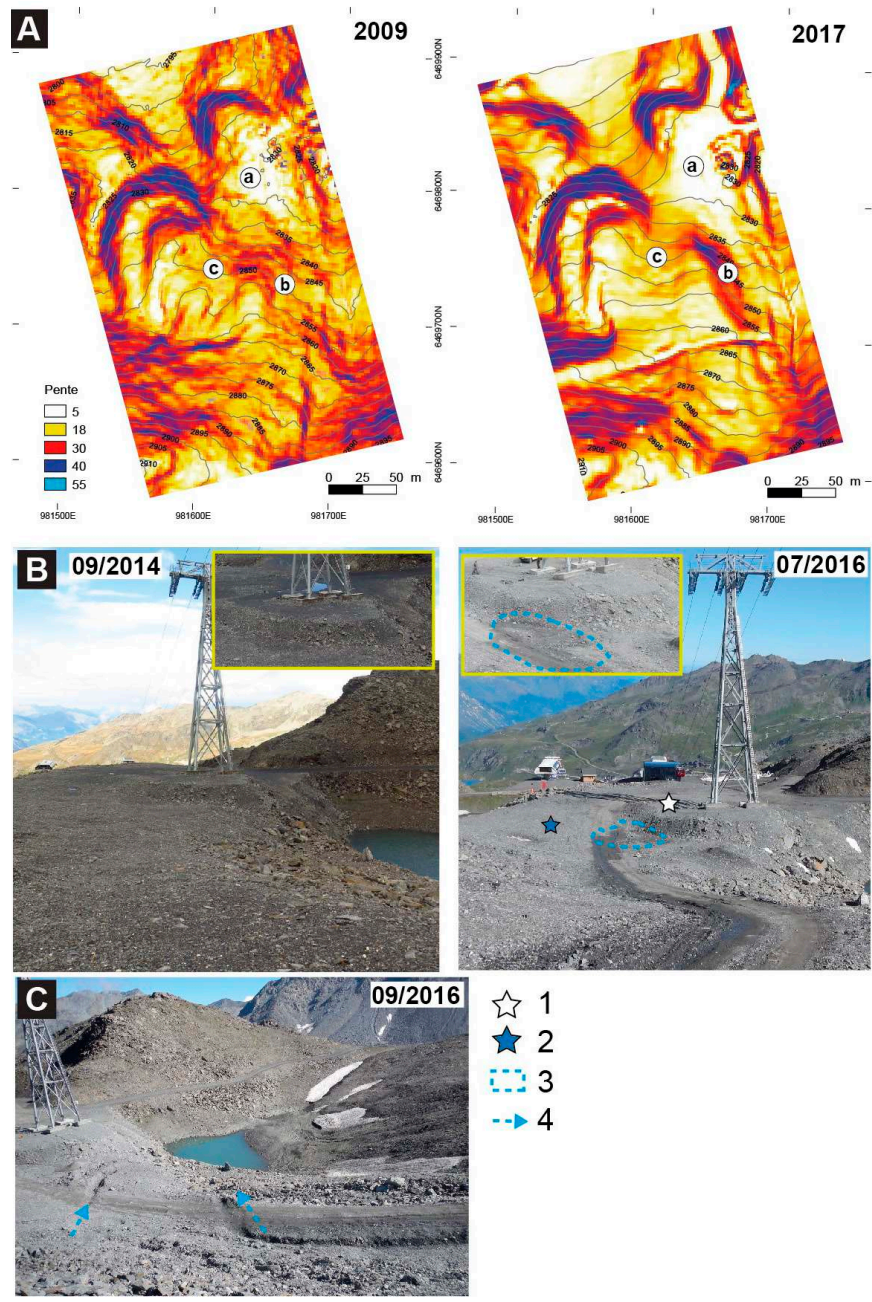

Fig. 6 - Évolution de la surface du glacier rocheux et modification des écoulements de surface entre 2009 et 2017.

A : Pente moyenne du glacier rocheux en 2009 et 2017. B : Évolution des écoulements de surface et de la microtopographie en amont de la plateforme du pylône avant (2014) et après (2016) la construction de la piste pour véhicule. C : Fossé creusé en août 2016 pour détourner les écoulements de la piste pour véhicules. a : Aplanissement général du terrain autour de la plateforme du pylône ; $b$ : Augmentation de l'angle de pente en amont du pylône (de 30 à $40^{\circ}$ ); c : Réduction de la microtopographie (rugosité) par nivellement pour l'aménagement de la piste de ski. 1. Forage B1 ; 2. Forage B2 ; 3. Zone d'infiltration identifiée en juillet 2016; 4. Fossé et renvois d'eau creusés début août 2016 pour détourner les écoulements de surface.

Fig. 6 - Evolution of the surface of the rock glacier and modification of the surface flows between 2009 and 2017.

A: Slope angle of the rock glacier in 2009 and 2017. B: Evolution of surface flows and microtopography upstream of the pylon platform before (2014) and after (2016) the construction of the vehicle track. C: Ditch dug in August 2016 to divert runoff from the runway for vehicles. a: General flattening of the ground around the plat form of the pylon; $b$ : Increase of the slope angle upstream of the pylon (from 30 to $40^{\circ}$ ); c: Reduction of microtopography (roughness) by leveling for the development of the ski slope. 1. Borehole B1; 2. Borehole B2; 3. Infiltration zone identified in July 2016; 4. Ditch and water channel dug in early August 2016 to divert surface runoff.
La comparaison des données stratigraphiques et des profils ERT inversés permet d'aller plus loin dans les interprétations, à la fois en ce qui concerne la distribution des sédiments gelés et l'épaisseur des structures.

Le premier forage (B1), directement réalisé dans le secteur affecté par la subsidence et à proximité immédiate du pylône révèle certaines caractéristiques de la zone instable dont le type de matériaux présent sous les massifs des fondations déstabilisées (fig. 8). La subsurface est composée d'un niveau de sédiments non gelés de $10 \mathrm{~m}$ d'épaisseur, dont une couche de $4 \mathrm{~m}$ de remblai en surface (plateforme du pylône), qui surmonte une couche de blocs et de gros cailloux de $5 \mathrm{~m}$ d'épaisseur, typique des glaciers rocheux. À $9,8 \mathrm{~m}$ de profondeur, apparaît une matrice fine très humide (présence d'eau liquide), avant d'atteindre le substratum rocheux à 10,4 $\mathrm{m}$ de profondeur. Le sondage B2, réalisé à l'Ouest du pylône n ${ }^{\circ} 2$, de l'autre côté de la piste pour véhicules, donne des indications sur les types de matériaux présents dans un secteur moins anthropisé du glacier rocheux. Jusqu'à $6,30 \mathrm{~m}$ de profondeur, de petits blocs représentatifs des matériaux régulièrement rencontrés dans la partie centrale d'un glacier rocheux (Springman et al., 2012) sont traversés. Au-delà, une couche de gros blocs dans une matrice de glace a été reconnue avant d'atteindre le substratum rocheux à $12,7 \mathrm{~m}$ de profondeur.

La comparaison entre le profil P2 et les données stratigraphiques permet une interprétation plus fine du déclenchement de l'instabilité (fig. 9). La zone de transition est assez large (environ $12 \mathrm{~m}$ en subsurface), probablement du fait de la circulation et de l'infiltration de l'eau entre les débris non gelés et/ou la fusion de la glace de pores entre les débris, tandis qu'elle n'est large que de $4 \mathrm{~m}$, entre 9 et $23 \mathrm{~m}$ de profondeur en raison de la présence du substratum rocheux. Ainsi, l'eau infiltrée a-t-elle pu lessiver les matériaux fins au niveau des fondations du pylône, conduisant au développement d'une subsidence thermokarstique par suffosion (Murton, 2009) et, par suite, au basculement du pylône du fait du déséquilibre des fondations.

\section{Discussion}

Lorsqu'on évoque des instabilités en zone de permafrost, la dégradation due au réchauffement atmosphérique est en général avancée comme facteur principal (Bodin et al., 2015). Les résultats des méthodes déployées dans cette étude, ont toutefois montré le rôle significatif de l'eau dans le déclenchement de l'instabilité. Nous discutons, ci-après, le rôle respectif de la dégradation du permafrost et des modifications anthropiques comme facteurs préparatoires au déclenchement de l'instabilité.

\subsection{Une dégradation " naturelle » et accentuée du permafrost}

Les investigations géoélectriques ainsi que des mesures expérimentales réalisées en laboratoire (Duvillard et al., 2018) ont montré que le permafrost présent dans le glacier rocheux de Thorens était de type tempéré, i.e., proche du point de fusion. Alors que le permafrost était déjà dans un état critique, l'été 2015 caniculaire et le mois de juin 2016 très pluvieux ont nécessairement encore accéléré la dégradation du permafrost dans la zone de transition, préparant ainsi l'instabilité. L'été 2015 fut le deuxième été le plus 


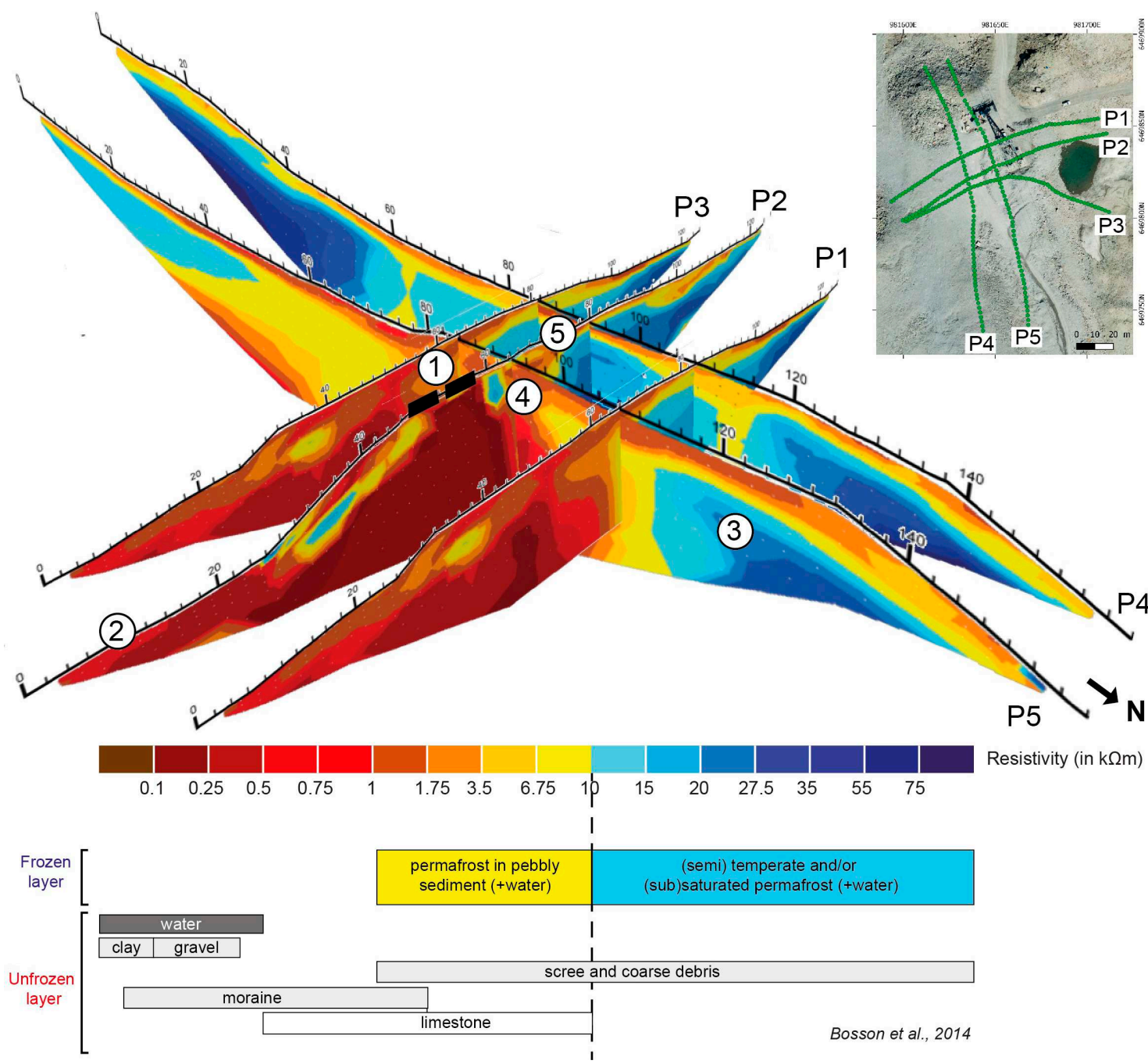

Fig. 7 - Bloc-diagramme en pseudo 3D des 5 profils ERT réalisés en septembre 2016. Interprétation selon l'échelle de Bosson et al. (2014).

1. Emplacement du pylône ; 2 . Lac ; 3. Front du lobe Est du glacier rocheux riche en glace; 4 . Subsidence ; 5 . Zone de transition entre les matériaux gelés et non-gelés.

chaud depuis 1959 dans les Alpes du Nord avec une température moyenne supérieure de $3,2^{\circ} \mathrm{C}$ par rapport à la normale 1961/1990 et de $2^{\circ} \mathrm{C}$ par rapport à la normale 1981/2010 (AGATE, 2018). Le mois de juin 2016 ne fut pas particulièrement chaud mais caractérisé par de fortes précipitations ; il fut le plus arrosé dans les Alpes du Nord depuis l'année 2000 avec un excédent de 50 \% (AGATE, 2018). Le fort cumul de précipitations du mois de juin 2016, qui s'additionne au déneigement précoce de la piste pour véhicules, a sans doute accéléré la fonte de la neige et donc intensifié les écoulements de surface.

Par ailleurs, les travaux de terrassement menés sur la surface du glacier rocheux, particulièrement dans la zone d'instabilité autour du pylône, ont très vraisemblablement conduit à un approfondissement de la couche active et à la dégradation locale du sommet de la couche gelée (i.e., le toit du permafrost) comme observé dans le forage B2 à $6,3 \mathrm{~m}$ de profondeur. Malgré une précision limitée dans l'alignement des nuages de points qui se traduit par une incertitude de l'ordre de $2 \mathrm{~m}$ au niveau de la différence des MNT (tab. 1), une différence d'altitude de 2 à $4 \mathrm{~m}$ au niveau du déblai de la zone d'instabilité est toutefois mesurée entre
Fig. 7 - Block diagram in pseudo $3 D$ of the 5 ERT profiles realized in September 2016.

1. Location of the pylon; 2. Lake; 3. Ice-rich East lobe of the rock glacier; 4. Subsidence; 5. Transition zone between frozen and non-frozen materials.

2009 et 2017 (fig. 5). Cette dégradation du permafrost n'est toutefois que fortement suggérée en l'absence d'une instrumentation permettant de comparer l'état thermique du permafrost avant et après l'aménagement du glacier rocheux.

\subsection{Le rôle prépondérant de l'eau}

Le rôle principal dans le déclenchement de l'instabilité du pylône peut être attribué à l'eau. Les observations ont montré que le nivellement et la compaction de la surface du glacier rocheux ont favorisé le ruissellement. La canalisation du ruissellement par la piste et son barrage par le remblai ont induit une infiltration préférentielle au droit des fondations du pylône. La déstabilisation du pylône serait donc due à l'effet combiné de deux phénomènes : (i) l'infiltration del'eau a pu abaisser localement le toit du permafrost. La grande profondeur de la couche active dans le forage B2, à 6,3 m, pourrait être due à ce phénomène. Dans d'autres glaciers rocheux actifs, les valeurs habituelles de profondeur de la couche active se situent en effet plutôt entre $2,5 \mathrm{~m}$, i.e., valeur mesurée en forage à Bellecombes par Bodin et al. (2015), et 3,5 m, i.e., valeurs issues 


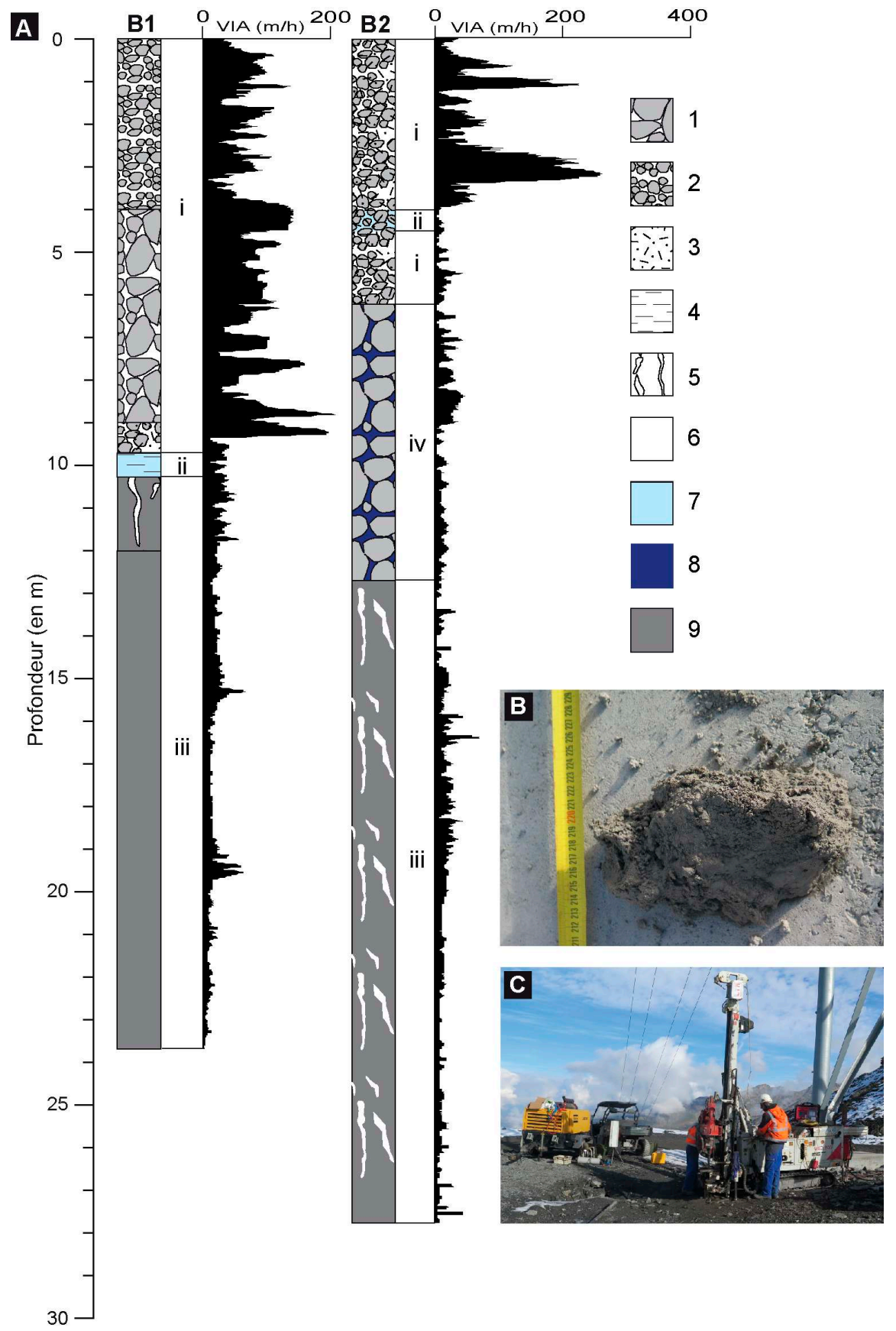

Fig. 8 - Formations rencontrées à l'occasion de deux forages mis en œuvre à proximité du pylône $\mathrm{n}^{\circ} 2$.

A : Stratigraphies interprétées des forages B1 et B2 avec la Vitesse Instantanée d'Avancement (en $\mathrm{m}^{-\mathrm{h}^{-1}}$. $\mathrm{B}$ : Matrice d'argile identifiée en $\mathrm{B} 1$ à $9,8 \mathrm{~m}$ de profondeur. $\mathrm{C}$ : Réalisation du forage B1. 1. Blocs moyens à petits $(10$ à $30 \mathrm{~cm}) ; 2$. Petit blocs et cailloux ; 3 . Graviers et sables ; 4. Matrices fines humides (argiles ?) ; 5. Fractures : 6. Vide (air) ; 7. Eau ; 8. Glace de pores (lentille de glaces ?) ; 9. Substratum rocheux ; i : Sédiments non gelés ; ii : Eau ; iii : Substratum rocheux ; iv : Sédiments gelés ; $\mathrm{v}$ : Substratum rocheux gelé.

Fig. 8 - Layers observed from the two boreholes drilled near the pylon \#2.

A: Stratigraphy of the boreholes $B 1$ and $B 2$ with the instantaneous speed of penetration (en m. $h^{-1}$ ). B: Clay matrix identified in $B 1$ at $9.8 \mathrm{~m}$ in depth. C: Borehole B1. 1. Boulders; 2. Boulders and cobbles (10 to $30 \mathrm{~cm}$ ); 3. Gravels and sands; 4. Wet fine matrices (clays ?); 5. Cracks; 6. Voids (air); 7. Water; 8. Pore ice (ice lens ?); 9. Bedrock; i: Unfrozen sediments; ii: Water; iii: Bedrock; iv: Frozen sediments; v: Frozen bedrock.

de mesures géophysiques ou forages instrumentés réalisés par Bodin et al., (2016) et Kenner et al. (2017) ; (ii) la circulation de l'eau dans les matériaux dégelés a pu lessiver la matrice fine, comme le laisse présager l'absence de matrice dans les couches de subsurface $\mathrm{du}$ forage $\mathrm{B} 1$, induisant un phénomène de suffosion. L'entonnoir et les fractures concentriques observées dans la zone de subsidence à l'ouest du pylône en seraient les marques de surface.

\subsection{Préconisations et solutions de confortement}

Étant donné que la présence de permafrost avait été vérifiée, se pose la question de savoir si la déstabilisation du pylône aurait pu être évitée. Le rapport géotechnique préalable avait préconisé un déplacement du pylône vers l'amont, dans une zone semblant présenter moins de glace, ainsi que la mise en place d'un massif de cailloux à forte porosité pour favoriser le refroidissement du terrain sous-jacents par les circulations d'air. Le pylône a en outre été construit avec une base ajustable dans le sens latéral de la pente. Ces deux dernières mesures sont conformes aux préconisations données par Bommer et al. (2010).

L'étude préalable était toutefois fondée uniquement sur des mesures géophysiques. Un forage de reconnaissance aurait montré que le substratum rocheux n'était situé qu'à une dizaine de mètres de profondeur seulement, et aurait peut-être conduit à concevoir une fondation sur pieux ancrés sur le rocher. Le rapport ne contenait par ailleurs aucune préconisation quant à la maîtrise des eaux de surface. Celles-ci sont en effet généralement absentes à la surface des glaciers rocheux non anthropisés. Il s'ensuit que les travaux de terrassement et de construction des pistes ont été effectués sans étude préalable de leur impact sur le ruissellement. 


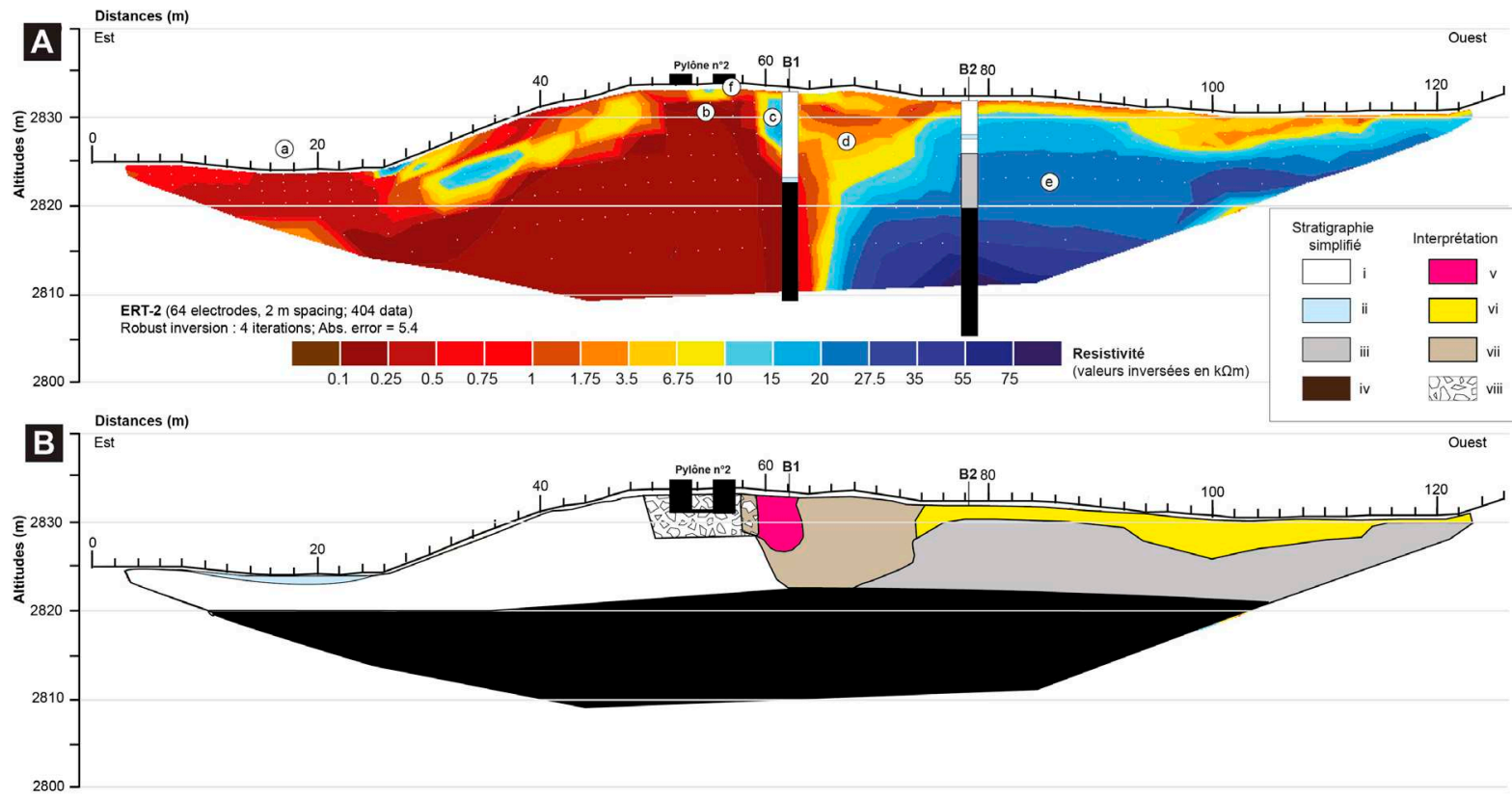

Fig. 9 - Coupe 2D transversal Est-Ouest de la zone instable du glacier rocheux (sous le pylône).

A : Comparaison du profil de tomographie électrique $\mathrm{P} 2$ et des profils stratigraphiques issus des forages B1 et B2. B : Schéma de synthèse des informations. i : Sédiments non gelés; ii : Eau; iii : Sédiments gelés; iv : Substratum rocheux; v: Subsidence; vi : Couche active ; vii : Zone de transition entre sédiments gelés/non gelés ; viii : Graviers ; a : Lac $\mathrm{b}$ : Graviers sous les fondations ; $\mathrm{:} \mathrm{Subsidence} \mathrm{;} \mathrm{d}$ : Zone de transition avec tranche de débris rocheux et glace; e : Permafrost.

Finalement, ce sont bien des pieux ancrés sur le rocher qui ont dû être mis en œuvre pour stabiliser le pylône.

Les fondations du pylône ont été ancrées sur le substratum rocheux au cours de l'été 2017 à l'aide de 38 micropieux de $17 \mathrm{~m}$ de long afin de garantir une stabilité en cas de nouvel affaissement de l'ensemble des matériaux de la zone d'instabilité. Ces travaux très coûteux (environ $1 \mathrm{M} €$ ) représentent $7 \%$ de l'investissement de départ seulement cinq ans après la construction. Par ailleurs, la piste pour véhicules a été déplacée en octobre 2017 dans la partie centrale du glacier rocheux afin que la zone située en amont du pylône canalise moins les écoulements de surface. Malgré le coût important de construction comme de confortement, aucun forage n'a été instrumenté de manière permanente pour suivre les déformations et/ou les températures en profondeur.

\subsection{Représentativité du glacier rocheux de Thorens}

L'évolution du glacier rocheux de Thorens n'est pas représentative de celle des glaciers rocheux de la région en raison de l'anthropisation très marquée de celui-ci. Parmi les 3261 glaciers rocheux (814 actifs, 671 inactifs, et 1776 fossiles) recensés dans les Alpes françaises (Marcer et al., 2017), seule une dizaine est aménagée (présence de pistes de ski et/ou de remontées mécaniques) (Duvillard et al., soumis). Ces glaciers rocheux aménagés sont généralement caractérisés par une faible pente et ont souvent subi des modifications importantes par nivellement de leur surface pour l'aménagement de pistes de ski. Seuls deux autres cas ont fait l'objet d'études scientifiques détaillées en relation avec des déstabilisations d'éléments d'infrastructures (Duvillard et al., sous presse). Aux
Fig. 9 - 2D cross section East-West of the unstable zone of the rock glacier under the pylon.

A: Comparison of the P2 electrical tomography profile and stratigraphic profiles from $B 1$ and B2 boreholes. B: Synthesis sketch; i: Unfrozen sediments; ii: Water; iii: Frozen sediments; iv: Bedrock; v: Subsidence; vi: Active layer; vii: Transition area between unfrozen/frozen sediments; viii: Gravels. a: Lake; b: Gravels under the foundations; $c$ : Subsidence; $d$ : Transition area with sediments and ice; e: Permafrost.

Deux-Alpes, la gare d'arrivée du télésiège de Bellecombes (2 $710 \mathrm{~m})$, implantée sur un glacier rocheux, a dû être reprise en sous-œuvre en 2013, suite à son affaissement. Comme pour le pylône $n^{\circ} 2$ du funitel de Thorens, aucun forage de reconnaissance n'avait été réalisé et la gare n'avait pas été ancrée sur le rocher, mais posée sur une dalle flottante après excavation d'une couche de glace. Là aussi, les forages de recherche réalisés à proximité ont révélé que le rocher n'aurait été qu'à une dizaine de mètres de profondeur (Bodin et al., 2010). À Orelle, cinq pylônes du télésiège de Plan Bouchet sont implantés sur un glacier rocheux. Les pylônes $n^{\circ} 7,8$, et 9 ont dû être reconstruits récemment, à deux reprise en 2011 et 2018. Comme à Val Thorens, des terrassements pour pistes de ski ont été réalisés à proximité. Dans ces deux cas toutefois, le ruissellement ne semble pas avoir joué de rôle déclencheur de l'instabilité. Les cas des Deux-Alpes comme de Val Thorens montrent que les études de géophysique ne sont pas suffisantes pour des implantations, et devraient systématiquement être accompagnées d'un forage de reconnaissance. Il est en effet connu que la méthode ERT tend à surestimer l'épaisseur du permafrost et ne permet pas de reconnaître de façon fiable la profondeur du toit rocheux.

\section{Conclusion}

La rétro-analyse multi-méthodes de la déstabilisation du pylône $\mathrm{n}^{\circ} 2 \mathrm{du}$ funitel de Thorens a permis d'identifier différents facteurs préparatoires à l'instabilité du pylône survenue en juillet 2016. (i) un choix risqué dans d'implantation en 2011 du pylône sur un glacier rocheux et sans aucun suivi de l'ouvrage ni des conditions thermiques sur le long terme, avec un terrassement de 2 à $6 \mathrm{~m}$ de 
la surface naturelle du glacier rocheux ; (ii) une modification et une concentration des écoulements d'eau vers le pylône, favorisant l'infiltration des eaux et le lessivage des matériaux sous les fondations. La suffosion constituerait ainsi le facteur principal de déclenchement de l'instabilité. L'eau représente également très probablement un facteur local d'accélération de la dégradation du permafrost, en particulier en raison d'un état thermique très proche du point de fusion et des intenses précipitations du mois de juin 2016.

La réalisation de forages de reconnaissance dans la phase d'étude aurait sans doute conduit à concevoir des fondations ancrées sur le substratum rocheux. Cette solution a finalement dû être mise en œuvre au cours de l'été 2017 avec la reprise en sous-œuvre de 38 micropieux de $17 \mathrm{~m}$ de long afin de garantir une stabilité satisfaisante du pylône. Ces travaux très coûteux de confortement (7\% de l'investissement de départ) auraient sans doute pu être évités. Les glaciers rocheux étant particulièrement sensibles à l'augmentation des températures de l'air et aux modifications anthropiques fortes telles que des terrassements, la construction d'infrastructures en haute montagne nécessite des études préalables multi-méthodes suivies de préconisations en termes d'adaptabilité des fondations et de suivi des conditions thermiques du terrain.

\section{Remerciements}

Les auteurs remercient la SETAM (Société d'Exploitation des Téléphériques Tarentaise Maurienne) et le bureau d'étude SAGE (Société Alpine de Géotechnique) pour la collaboration mise en place et toutes les informations géotechniques fournies. Nous remercions K. Génuite, J. Berthet (société Styx 4D), G. Heller et A. Ollive pour leur aide sur le terrain et pour le traitement de certaines données. Nous remercions A. Revil, G. Ménard et X. Bodin ainsi que les deux relecteurs anonymes pour les riches commentaires et suggestions sur ce travail. P-A. Duvillard a bénéficié d'une allocation de thèse financée par Ingénierie des Mouvements du Sol et des Risques Naturels (IMSRN) et l'Association Nationale de la Recherche et de la Technologie (ANRt). Cette étude s'intègre dans le projet FEDER POIA PermaRisk.

P.-A. Duvillard a élaboré le travail de recherche, réalisé les mesures de terrain, traité les données et écrit la version initiale de l'article, sous la direction de L. Ravanel et P. Schoeneich. LR., PS. et M. Marcer ont contribué à l'amélioration du manuscrit. M.M. a également participé à l'acquisition de données sur le terrain. J.F. Piard a apporté un soutien logistique et des données inédites.

\section{Références}

AGATE (2018) - Agence Alpine des Territoires, https://agateterritoires.fr, consulté en nov. 2018

Arenson L.U., Hoelzle M., Springman S. (2002) - Borehole deformation measurements and internal structure of some rock glaciers in Switzerland. Permafrost and Periglacial Process, 13, 117-135.

DOI : $10.1002 / p p p .414$

Arenson L.U., Phillips M., Springman S.M. (2009) - Geotechnical consideration and technical solutions for infrastructure in mountain permafrost. In Krugger M.I., Stern H.P. (Eds), New permafrost and glacier research. Hauppauge, NY, Nova, 3-50.

Arenson L.U. and Jakob M. (2017) - Permafrost-related geohazards and infrastructure construction in mountainous environments. Oxford Research Encyclopedia of Natural Hazard Science, 30.

DOI : 10.1093/acrefore/9780199389407.013.292

Beniston M., Farinotti D., Stoffel M., Andreassen L. M., Coppola E., Eckert N., Fantini A., Giacona F., Hauck C., Huss M., Huwald H., Lehning M., López-Moreno J.-I., Magnusson J., Marty C., Morán-Tejéda E., Morin S., Naaim M., Provenzale A., Rabatel A., Six D., Stötter J., Strasser U., Terzago S., \& Vincent C. (2018) - The European mountain cryosphere: a review of its current state, trends, and future challenges. The Cryosphere, $12,759-794$.

DOI : $10.5194 / \mathrm{tc}-12-759-2018$

Bodin X, Desvarreux P, Fabre D, Krysiecki JM, Gay M, Marie R, Lorier L, Schoeneich P, Vallon M (2010) - Analyse des risques induits par la dégradation du permafrost alpin. ADRGT-PACTEGIPSA-lab-LGGE. Rapport final, Projet financé par la Fondation MAIF.

Bodin X., Deline P., Schoeneich P., Ravanel L., Magnin F., Krysiecki J.M., Echelard T. (2015) - Le permafrost de montagne et les processus géomorphologiques associés : évolutions récentes dans les Alpes françaises. Revue de Géographie Alpine, 103-2.

DOI : $10.4000 /$ rga.2806

Bodin X., Krysiecki J.M., Schoeneich P., Le Roux O., Lorier L., Echelard T., Peyron M., Walpersdorf A. (2016) - The 2006 Collapse of the Bérard Rock Glacier (Southern French Alps). Permafrost and Periglacial Process, 28, 209-223.

DOI : $10.1002 /$ ppp.1887

Bodin X., Thibert E., Sanchez O., Rabatel A., and Jaillet S. (2018) Multi-Annual Kinematics of an Active Rock Glacier Quantified from Very High-Resolution DEMs: An Application-Case in the French Alps. Remote Sensing, 10, 547.

DOI : $10.3390 / \mathrm{rs} 10040547$

Bommer C., Phillips M., Arenson L.U. (2010) - Practical recommendations for planning, constructing and maintaining infrastructure in mountain permafrost. Permafrost Periglaclacial Processes, 21, 97-104.

DOI : $10.1002 /$ ppp.679

Bosson JB., Deline P., Bodin X., Schoeneich P., Baron L., Gardent M. \& Lambiel C. (2014) - The influence of ground ice distribution on geomorphic dynamics since the Little Ice Age in proglacial areas of two cirque glacier systems. Earth Surface Processes and Landforms, 40, 666-680.

DOI : $10.1002 /$ esp.3666

Capt M., Bosson J.-B., Fischer M., Micheletti N. \& Lambiel C. (2016) - Decadal evolution of a very small heavily debriscovered glacier in an Alpine permafrost environment. Journal of Glaciology, 62, 535-551.

DOI : $10.1017 /$ jog.2016.56

Cicoira A., Vieli A. \& Faillettaz J. (2018) - Investigating the influence of temperature and liquid water on variations in rockglacier flow. Book of Abstracts of the $5^{\text {th }}$ European Conference On Permafrost, 390, 2p.

Dall'Asta E., Forlani G., Roncella R., Santise M., Diotri F. \& Morra di Cella U. (2017) - Unmanned Aerial Systems and DSM matching 
for rock glacier monitoring. ISPRS Journal of Photogrammetry and Remote Sensing, 127, 102-114.

DOI : 10.1016/j.isprsjprs.2016.10.003

Dahlin T., Zhou B. (2004) - A numerical comparison of 2D resistivity imaging with 10 electrode arrays. Geophysical Prospecting, 52, 379-398.

DOI : 10.1111/j.1365-2478.2004.00423.x

Debelmas J. (1989) - Notice explicative de la carte géologique de Modane à 1/50000. Orléans: BRGM.

Delaloye R., Lambiel C., Gärtner-Roer I. (2010) - Overview of rock glacier kinematics research in the Swiss Alps. Geographica Helvetica, 65, 135-145.

DOI : $10.5194 /$ gh-65-135-2010

Dobiński W. (2011) - Permafrost. Earth-Science Reviews, 108, 158-169.

DOI : 10.1016/j.earscirev.2011.06.007

Duvillard P.A., Ravanel L., Deline P. (2015) - Risk assessment of infrastructure destabilisation due to global warming in the high French Alps. Revue de géographie alpine, 103-2.

DOI : $10.4000 /$ rga.2896

Duvillard P.A., Revil A., Qi Y., Soueid Ahmed A., Coperey A. and Ravanel L. (2018) - Three-Dimensional electrical conductivity and induced polarization tomography of a rock glacier. Journal of Geophysical Research: Solid Earth, 123.

DOI : 10.1029/2018JB015965

Duvillard P.A., Ravanel L., Marcer M. and Schoeneich P. (2019) Recent evolution of damage to infrastructure on permafrost in the French Alps. Regional Environmental Change.

DOI : 10.1007/s10113-019-01465-z

Duvillard P.A., Ravanel L., Schoeneich P., Deline P., Marcer M. and Magnin F. (soumis) - Risk assessment and adaptation strategies for infrastructure on permafrost in the French Alps. Mountain Research and Development.

Fabre D., Cadet H., Lorier L., and Leroux O. (2015) - Detection of Permafrost and Foundation Related Problems in High Mountain Ski Resorts. In: Lollino G., Manconi A., Clague J., Shan W., Chiarle M. (Eds), Engineering Geology for Society and Territory, vol. 1, 321-324.

DOI : 10.1007/978-3-319-09300-0_60

Gardent M., Rabatel A., Dedieu J.-P., Deline P. (2014) Multitemporal glacier inventory of the French Alps from the late 1960s to the late 2000s. Global and Planetary Change, 120, 24-37. DOI : 10.1016/j.gloplacha.2014.05.004.

Girardeau-Montaut D. (2006) - Détection de changement sur des données géométriques tridimensionnelles. Thèse de doctorat, Télécom ParisTech.

Guillot F. (1982) - La vallée du Ponturin (Vanoise septentrionale, Alpes françaises). Mémoire de Géologie appliquée. L'université des sciences et techniques de Lille.

Haeberli W. (1992) - Construction, environmental problems and natural hazards in periglacial mountain belts. Permafrost and Periglacial Processes, 3, 111-124.

DOI : $10.1002 /$ ppp.3430030208

Haeberli W., Hallet B., Arenson L.U., Elconin R., Humlum O., Kääb A., Kaufmann V., Ladanyi B., Matsuoka N., Springman S., Vonder Mühll D. (2006) - Permafrost creep and rock glacier dynamics. Permafrost and Periglacial Processes, 17 (3), 189-214.
DOI : $10.1002 /$ ppp.561

Harris C., Arenson L.U., Christiansen H.H., Etzelmüller B., Frauenfelder R., Gruber S., Haeberli W., Hauck C., Hölzle M., Humlum O., Isaksen K., Kääb A., Kern-Lütschg M.A., Lehning M., Matsuoka N., Murton J.B., Nötzli J., Phillips M., Ross N., Seppälä M., Springman S.M., Vonder Mühll D. (2009) Permafrost and climate in Europe: Monitoring and modelling thermal, geomorphological and geotechnical responses. EarthScience Reviews, 92, 117-171.

DOI : 10.1016/j.earscirev.2008.12.002

Ikeda A., Matsuoka N., \& Kääb A. (2008) - Fast deformation of perennially frozen debris in a warm rock glacier in the Swiss Alps: an effect of liquid water. Journal of Geophysical Research, 113, F01021.

DOI : $10.1029 / 2007$ JF000859

Jones D.B., Harrison S., Anderson K., Whalley W.B. (2019) Rock glaciers and mountain hydrology: A review. Earth-Science Reviews 193, 66-90.

DOI : 10.1016/j.earscirev.2019.04.001

Kääb A., Frauenfelder R. \& Roer I. (2007) - On the response of rockglacier creep to surface temperature increase. Global and Planetary Change, 56, 172-187.

DOI : 10.1016/j.gloplacha.2006.07.005

Kenner R., Bühler Y., Delaloye R., Ginzler C. \& Phillips M. (2014) Monitoring of high alpine mass movements combining laser scanning with digital airborne photogrammetry. Geomorphology, 206, 492-504

DOI : 10.1016/j.geomorph.2013.10.020

Kenner R., Phillips M., Beutel J., Hiller M., Limpach P., Pointner E., \& Volken M. (2017) - Factors Controlling Velocity Variations at Short-Term, Seasonal and Multiyear Time Scales, Ritigraben Rock Glacier, Western Swiss Alps. Permafrost and Periglacial Processes, 28, 675-684.

DOI : $10.1002 /$ ppp.1953

Kneisel C. (2006) - Assessment of subsurface lithology in mountain environments using 2D resistivity imaging. Geomorphology, 80, 32-44.

DOI : 10.1016/j.geomorph.2005.09.012

Kneisel C., Hauck C., Fortier R., \& Moorman B. (2008) - Advances in geophysical methods for permafrost investigations. Permafrost and Periglacial Processes, 19 (2), 157-178.

DOI : $10.1002 /$ ppp.616

Krainer K., Bressan D., Dietre B., Haas J.N., Hajdas I., Lang K., Mair V., Nickus U., Reidl D., Thies H., Tonidandel D. (2015) A 10,300-year-old permafrost core from the active rock glacier Lazaun, southern Ötztal Alps (South Tyrol, northern Italy). Quaternary Research 83, 324-335.

DOI : 10.1016/j.yqres.2014.12.005

Kummert M., Delaloye R., \& Braillard L. (2017) - Erosion and sediment transfer processes at the front of rapidly moving rock glaciers: Systematic observations with automatic cameras in the western Swiss Alps. Permafrost and Periglacial Processes, 29 (1), 21-33.

DOI : $10.1002 /$ ppp. 1960

Lambiel C., Maillard B., Martin S., Pellitero Ondicol R., Schoeneich P., Reynard E. (2013) - Adaptation of the geomorphological mapping system of the University of Lausanne 
for ArcGIS. $8^{\text {th }}$ International Conference on Geomorphology (IAG), Paris, 27- 31 Aug. 2013.

Loke M.H., Barker R.D. (1996) - Rapid least-squares inversion of apparent resistivity pseudosections by a quasi-Newton method1. Geophysical Prospecting, 44, 131-152.

DOI : 10.1111/j.1365-2478.1996.tb00142.x

Luethi R., Phillips M., Lehning M. (2017) - Estimating nonconductive heat flow leading to intra-permafrost talik formation at the Ritigraben Rock Glacier (Western Swiss Alps): Estimating non-Conductive Heat Flow. Permafrost Periglacial Process, 28, 183-194.

DOI : $10.1002 /$ ppp.1911

Lugon R., \& Stoffel M. (2010) - Rock-glacier dynamics and magnitude-frequency relations of debris flows in a high-elevation watershed: Ritigraben, Swiss Alps. Global and Planetary Change, 73 (3-4), 202-210.

DOI : 10.1016/j.gloplacha.2010.06.004

Magnin F., Deline P., Ravanel L., Noetzli J., Pogliotti P. (2015) Thermal characteristics of permafrost in the steep alpine rock walls of the Aiguille du Midi (Mont Blanc Massif, 3842 m a.s.l.). The Cryosphere 9, 109-121.

DOI : $10.5194 /$ tc-9-109-2015

Marcer M., Bodin X., Brenning A., Schoeneich P., Charvet R., Gottardi F. (2017) - Permafrost Favorability Index: Spatial Modeling in the French Alps Using a Rock Glacier Inventory. Frontiers in Earth Science, 5:105.

DOI : $10.3389 /$ feart.2017.00105

Marcer, M., Serrano, C., Brenning, A., Bodin, X., Goetz, J., and Schoeneich, P. (2019) - Evaluating the destabilization susceptibility of active rock glaciers in the French Alps. The Cryosphere, 13, 141-155,

DOI : $10.5194 /$ tc-13-141-2019

Maurer H., \& Hauck C. (2007) - Geophysical imaging of alpine rock glaciers. Journal of Glaciology, 53 (180), 110-120.

DOI : $10.3189 / 172756507781833893$

Murton J.B. (2009) - Global Warming and Thermokarst, Permafrost Soils. Springer, Berlin Heidelberg, pp.185-203.

PERMOS (2016) - Permafrost in Switzerland 2010/2011 to 2013/2014. Noetzli J, Luethi R, Staub B (eds.), Glaciological Report Permafrost No. $12-15$ of the Cryospheric Commission of the Swiss Academy of Sciences, $85 \mathrm{pp}$.

Pomerleau F., Colas F., Siegwart R., and Magnenat S. (2013) Comparing ICP variants on real-world data sets. Autonomous Robots, 34, 133-148.

DOI : $10.1007 / \mathrm{s} 10514-013-9327-2$

Phillips M., Ladner F., Müller M., Sambeth U., Sorg J., Teysseire P. (2007) - Monitoring and reconstruction of a chairlift midway station in creeping permafrost terrain, Grächen, Swiss Alps. Cold Regions Science and Technology, 47, 32-42.

DOI : 10.1016/j.coldregions.2006.08.014

Ravanel L., Duvillard P.-A., Jaboyedoff M., Lambiel C. (2018) Recent evolution of an ice-cored moraine at the Gentianes Pass, Valais Alps, Switzerland. Land Degradation and Development, 29, 3693-3708.

DOI : $10.5194 /$ esurf-4-103-2016
DOI : 10.1002/ldr.3088

Roer I., Haeberli W., Avian M., Kaufmann V., Delaloye R., Lambiel C. \& Kääb A. (2008) - Observations and considerations on destabilizing active rock glaciers in the European Alps. Ninth International Conference on Permafrost, (4), 1505-1510.

DOI : $10.5167 /$ uzh-6082

Scapozza C., Lambiel C., Gex P., Reynard E. (2011a) - Prospection géophysique multi-méthodes du pergélisol alpin dans le sud des Alpes suisses. Géomorphologie: Relief, Processus, Environnement, 17 (1), 15-32.

DOI : $10.4000 /$ geomorphologie. 8765

Scapozza C., Lambiel C., Baron L., Marescot L., Reynard E. (2011b) - Internal structure and permafrost distribution in two alpine periglacial talus slopes, Valais, Swiss Alps. Geomorphology, 132 (3-4), 208-221.

DOI : $10.1016 /$ j.geomorph.2011.05.010

Scapozza C. and Laigre L. (2014) - The contribution of Electrical ResistivityTomography(ERT) in Alpine dynamicsgeomorphology: case studies from the Swiss Alps. Géomorphologie : Relief, Processus, Environnement, 20 (1), 27-42.

DOI : 10.4000/geomorphologie.10474

Scott W., Sellmann P. \& Hunter J. (1990) - Geophysics in the Study of Permafrost. In Geotechnical and Environmental Geophysics, 1, 355-384.

DOI : $10.1190 / 1.9781560802785 . c h 13$

Schoeneich P., Dall'Amico M., Deline P., Zischg A. (2011) - Hazards related to permafrost and to permafrost degradation. PermaNET project, state-of-the-art report 6.2. On-line publication ISBN 9782-903095-59-8

Schoeneich P., Krysiecki J.M., Mingrat L., Huwald H. (2015) - DTS ground temperature measurements in mountain permafrost - the 2Alpes-3065 borehole (French Alps). GeoQuebec, 823, 6 p.

Schoeneich P. \& Ribeyre C. (2017) - Commune de Lanslevillard (73) Etude géomorphologique et géophysique du glacier rocheux du Col du Lou suite à la lave torrentielle du 14 août 2015. Rapport non publié 1-42.

Smith M.W., Carrivick J.L. and Quincey D.J. (2016) - Structure from motion photogrammetry in physical geography. Progress in Physical Geography, 40, 247-275.

DOI : $10.1177 / 0309133315615805$

Springman S.M., Arenson L.U., Yamamoto Y., Maurer H., Kos A., Buchli T., \& Derungs G. (2012) - Multidisciplinary investigations on three rock glaciers in the Swiss Alps: legacies and future perspectives. Geografiska Annaler: Series A. Physical Geography, 94 (2), 215-243.

DOI : 10.1111/j.1468-0459.2012.00464.x

Stoffel M., Tiranti D., and Huggel C. (2014) - Climate change impacts on mass movements - case studies from the European Alps. Science of the Total Environment. 493, 1255-1266.

DOI : 10.1016/j.scitotenv.2014.02.102

Wirz V., Gruber S., Purves R. S., Beutel J., Gärtner-Roer I., Gubler S., and Vieli A. (2016) - Short-term velocity variations at three rock glaciers and their relationship with meteorological conditions. Earth Surface Dynamics, 4, 103-123. 
Sigles

ERT : tomographie électrique résistive

MNT : Modèles Numérique de Terrain

IGN : Institut Géographique National

PCS : Points de Contrôle au Sol

PFI : carte d'indice de présence de permafrost dans les Alpes Françaises

RMS : moyenne quadratique

TLS : balayage laser terrestre

UAV : drone

\section{Acronyms}

DEM: Digital Elevation Model

ERT: Electrical Resitivity Tomography

GCP: Ground Control Point

PFI: Permafrost Favourability Index

RMS: Root Mean Square error

TLS: Terrestrial Laser Scanning

UAV: Unmanned Aerial Vehicle

\section{Abridged English Version}

In the current context of climate change, high altitude rock slopes and surficial deposits in the European Alps are affected by geomorphological processes partly conditioned by permafrost degradation (Harris et al., 2009). Warming permafrost and high water content in frozen grounds are at the origin of an increasing risk of destabilization for infrastructure built in high mountain areas (e.g., huts and ropeway transport systems). The Val Thorens ski resort (Savoie, France) has more than 79 infrastructure elements related to ropeway transport systems located in permafrost-affected areas (fig. 1). During the last decade, three of them (two top stations and a pylon), built at an altitude of around 3,000 $\mathrm{m}$ a.s.l., experienced a partial destabilization requiring major reinforcement and stabilization work (Duvillard et al., in press). This article aims to understand the destabilization of the Thorens funitel pylon \#2 that occurred during the Summer 2016. The pylon was built in 2011 on an ice-rich rock glacier which suffered from severe anthropic alterations (earthwork) for the development of a ski slope and a vehicle track (Fabre et al., 2015) (fig. 2-3). The rock glacier is divided into two different lobes. Three evidences of geomorphological processes were identified after the instability (fig. 4): (i) subsidence of metric radius close to the foundations, (ii) opening of cracks upstream of the foundations, and (iii) surface runoff infiltrating the foundations upstream of the pylon platform. A multi-approach analysis was conducted to address two main concerns: (i) the presence or absence of permafrost in the rock glacier and its possible contribution in triggering the instability, (ii) the role of earthwork on the thermal regime of the rock glacier. Four methods have been used to document and identify the origins of the destabilization: (i) the reconstruction of surface geomorphological changes by comparing Digital Elevation Models obtained by aerial photogrammetry based on historical and recent imagery, (ii) the comparison of field photographs of different dates, (iii) an Electrical Resistivity Tomography campaign with 5 profiles in pseudo-3D to highlight contrasts and anomalies in ground resistivity, and (iv) two destructive drillings, 27.8 and $23.7 \mathrm{~m}$ deep, to validate the depth of the bedrock to provide a geotechnical solution. DEMs from 2009 and 2017 were obtained by using the Structure from Motion approach on historical airborne imagery and recent UAV photography (Smith et al., 2016), allowing to observe surface elevation slope variations (fig. 6). The setting up of the ski slope and the track for vehicles largely modified the micro-topography of the rock glacier as well as the surface runoff. The earthwork on the rock glacier concentrated the runoff by channelling the water upstream of the pylon platform. The 5 pseudo-3D ERT profiles (P1 and P2 respectively in figure 5) indicated high resistivity areas, reaching 10 to $55 \mathrm{k} \Omega \mathrm{m}$ (fig. 7). These values confirmed the presence of ice filling the soil pores in a temperate and water sub-saturated permafrost environment (Bosson et al., 2014). Although the foundations of the pylon \#2 are likely located in a permafrost-free area of the rock glacier, the pylon is located only $5 \mathrm{~m}$ East of a transition zone to an ice-rich permafrost where the subsidence occurred. This transition area is $12 \mathrm{~m}$-wide, possibly due to underground water infiltration and circulation at the interface between frozen and unfrozen materials, probably induced the leaching of the finest materials.

Borehole B1, located in the subsidence near the pylon showed that the rock glacier internal structure consisted of several ice-free layers. The first layer corresponds to $10 \mathrm{~m}$ of unfrozen sediments (fig. 8) while a fine wet matrix appeared at a depth of $9.8 \mathrm{~m}$ (presence of liquid water), before reaching the bedrock at a depth of $10.4 \mathrm{~m}$. Borehole B2, at the West side of the vehicle track, provided information on the type of the material that is present in a less anthropized zone of the rock glacier. The first $6 \mathrm{~m}$ consisted of small blocks representative of the central part of a rock glacier (Springman et al., 2012). From a depth of $6.3 \mathrm{~m}$, a layer of large blocks in an ice matrix was identified before reaching the bedrock at $12.7 \mathrm{~m}$. The internal structure of the rock glacier is uncommon (fig. 9). The East lobe of the rock glacier is divided into two zones: on the eastern part, rock material is unfrozen while, on the West, the soil seems to be frozen. The lake located on the East of the rock glacier, caused by a topographic barrier made by the bedrock, probably explains the absence of permafrost in the eastern part of the rock glacier (Kenner et al., 2017). Overall, results and field surveys/observations allowed to identify two principal factors causing the subsidence of the area close to the pylon during the Summer 2016. (i) the modification of the local hydrology, and (ii) the possible degradation of the permafrost body enhanced by the artificial alteration of the active layer and the water infiltration. The location of the infiltration zone may be due to (i) the presence of a transition zone between frozen and unfrozen grounds which may modify the permeability of the ground, and (ii) the topographical barrier corresponding to the pylon platform. The infiltrated water is suspected to wash out fine sediments at the base of the pylon platform causing ground subsidence by suffusion. In order to avoid water infiltration, a water line trench was dug in August 2016 to channelize the surface water flows towards the lake. The digital elevation models comparison revealed a significant smoothing of the rock glacier 
surface with embankment and 2 to 6 m-deep excavation (fig. 5). Debris moved from convex lobes to the frontal talus. This material filled natural depressions in order to achieve a homogenous gentle slope for skiing. The installation of the pylon and the earthwork critically altered the rock glacier surface and induces changes for the surface hydrology. Modifying the surface runoff caused significant underground infiltration and ground suffosion. This infiltration is considered to be the main triggering factor of the instability, as it is suspected to have initiated a local permafrost degradation. In order to avoid such events, it is suggested that permafrost characterisation is a critical point in foundation design. Surface smoothing in ice-rich permafrost sites should be avoided in order to not disrupt the active layer and the surface hydrology characteristics. 